\title{
On velocity and reactive scalar spectra in turbulent premixed flames
}

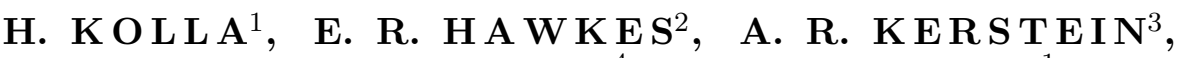 \\ N. SWA MINATHA N , and J. H. CHE N \\ ${ }^{1}$ Sandia National Laboratories, Livermore, CA 94550, USA \\ ${ }^{2}$ The Unversity of New South Wales, Sydney, Australia \\ ${ }^{3} 72$ Lomitas Road, Danville, CA 94526, USA \\ ${ }^{4}$ Engineering Department, University of Cambridge, Cambridge CB2 1PZ, UK
}

(Received 16 July 2014)

Kinetic energy and reactive scalar spectra in turbulent premixed flames are studied from compressible three-dimensional direct numerical simulations (DNS) of a temporally evolving rectangular slot-jet premixed flame, a statistically one-dimensional configuration. The flames correspond to a lean premixed hydrogen-air mixture at an equivalence ratio of 0.7 , preheated to $700 \mathrm{~K}$ and at 1 atmosphere and three DNS are considered with a fixed jet Reynolds number of 10000 and a jet Damköhler number varying between 0.13 and 0.54. For the study of spectra, motivated by the need to account for density change which can be locally strong in premixed flames, a new density-weighted definition for two-point velocity/scalar correlations is proposed. The density-weighted two-point correlation tensor retains the essential properties of its constant-density (incompressible) counterpart and recovers the density-weighted Reynolds-stress tensor in the limit of zero separation. The density weighting also allows the derivation of balance equations for velocity and scalar spectrum functions in the wavenumber space that illuminate physics unique to combusting flows; pressure-dilatation correlation is a source of kinetic energy at high wavenumbers and, analogously, reaction-rate-scalar fluctuation correlation is a high wavenumber source of scalar energy. These results are verified by the spectra constructed from the DNS data. The kinetic energy spectra show a distinct inertial range with a $-5 / 3$ scaling followed by a 'diffusive-reactive' range at higher wavenumbers. The exponential drop-off in this range shows a distinct inflection in the vicinity of the wavenumber corresponding to a laminar flame thickness, $\delta_{L}$, and this is attributed to the contribution from the pressure-dilatation term in the energy balance in wavenumber space. Likewise, a clear spike in spectra of major reactant species - hydrogen - arising from the reaction-rate term is observed at wavenumbers close to $\delta_{L}$. It appears that in the inertial range classical scaling laws for the spectra involving Kolmogorov scale are applicable but in the high wavenumber range where chemical reactions have a strong signature the laminar flame thickness produces a better collapse. It is suggested that a full scaling should perhaps involve Kolmogorov scale, laminar flame thickness, Damköhler number and Karlovitz number.

\section{Introduction}

The study of spectra of scalars is of great importance for a broad class of turbulent flows (Warhaft 2000; Dimotakis 2005): temperature and salinity fields in ocean flows, pollutant dispersion in atmospheric flows, magnetic fields in astrophysical flows, chemically reacting flows in industrial devices, to cite a few. In turbulent reacting flows, which 
are relevant in many industrial and engineering applications, stiff non-linear chemical reactions amongst a multitude of reacting scalars (tens to hundreds of chemical species) introduce length and time scales over a broad range. Compared to characteristic scales of the turbulent fluid flow the chemical scales are generally, except under extreme circumstances, at the finer end with some overlap. Nonetheless, the turbulence-scalar interaction is strong and two-way: fluid dynamics affects the scalar mixing which has a bearing on the finite rate chemical kinetics, while the reaction induced density change affects the fluid dynamics. With few notable exceptions, such as the spectral closure of EDQNM (Ulitsky \& Collins 1997; Xia et al. 2010), virtually every modelling methodology of turbulent reacting flows relies on some implicit or explicit assumptions about the dynamics of turbulence-scalar mixing and associated scalar spectra. For instance, the widely used laminar flamelet model for non-premixed turbulent combustion relies on representing the thermo-chemical manifold with a single scalar whose evolution equations employ models based on passive scalar mixing assumptions. While such assumptions are strictly valid for purely passive scalars in incompressible homogeneous isotropic turbulence, it is unclear what the consequences of variable density, finite rate chemical kinetics and differential species diffusion are on the evolution of scalar spectra. Recent studies (Swaminathan \& Grout 2006; Chakraborty \& Swaminathan 2007) have shown that scalar mixing physics are influenced by heat release in turbulent premixed flames, but their role in spectral behaviour is unclear. Indeed, even the assumption of universality of velocity spectra in the so called dissipation range maybe questionable for flows with strong local density change and dilatation, such as turbulent premixed flames, although it is routinely used for closing sub-grid Reynold's stresses in large eddy simulations (LES).

While spectra of passive scalars have been widely studied, spectra of reactive scalars have received limited attention. It is well known from Batchelor's early theory (Batchelor 1959) that passive scalars with large Schmidt number exhibit a $k^{-1}$ scaling of the power spectrum in the so-called viscous-convective wavenumber, $k$, range in which the spectral transfer of velocity is diffusive but that of the scalar is convective. On the other hand, scalars with small Schmidt number (Batchelor et al. 1959) exhibit an inertial-diffusive range with a $k^{-17 / 3}$ scaling for the scalar spectrum. Corrsin (1961) extended these scaling laws for the case of a scalar undergoing a first-order reaction and elucidated the influence of the reactivity on the scalar spectrum in the relevant wavenumber ranges for both large and small Schmidt numbers. However, Corrsin's analysis is restricted to a very dilute reactant whose dynamics do not affect the flow field. Nonetheless his results clearly show that the spectral dynamics of reactive scalar depend not just on scales of the background turbulent flow but also on the characteristic scales of the reactions. Similarly, G. Kosály (1993) examined a bi-variate reacting system in an incompressible turbulent mixing layer and derived the reacting scalar spectra in the slow and fast chemistry limits to compare against the measurements of Bilger et al. (1991). More recently Wang et al. (2007) and Vaishnavi et al. (2008) report dissipation spectra of mixture fraction, a conserved scalar, in turbulent non premixed flames from experiments and DNS, respectively. Knaus \& Pantano (2009) examined spectra of kinetic energy, mixture fraction and temperature from DNS of non-premixed reacting shear layers. They note that the influence of heat release on the spectra can be accounted for simply by considering density-weighted large-scale quantities in the classical scaling laws (except for the dissipation range of the temperature spectrum). Even though their results are based on DNS with single step chemical kinetics, they are very encouraging. They essentially confirm the arguments of Bilger (2004) who showed that for most practical flows turbulence will overwhelm dilatation due to heat release in non-premixed flames, and hence passive scalar behaviour is to be expected. This is, however, not true of premixed flames in which the local dilatation due 
to heat release is relatively stronger (Bilger 2004). Hence the spectral dynamics of both velocity and scalars in premixed flames are likely to be very different from those of nonpremixed flames. Furukawa et al. (2002) report kinetic energy spectra, conditioned on fresh and burnt gas fluids, for large Damköhler number turbulent premixed flames and observe an increase in kinetic energy as well as anisotropy in the burnt side relative to the unburnt side. To the best of our knowledge the only works that have reported reacting scalar spectra in turbulent premixed flames are the experimental works of Guttenfelder et al. (2003) and Kariuki et al. (2012) who report spectra of hydroxyl radical. Little is known of spectra and co-spectra of reacting scalars at realistic levels of heat release in premixed flames, as noted by Dimotakis (2005) and Knaus \& Pantano (2009), and this is the primary focus of the present study.

The study of spectra is made difficult by the fact that measuring spectra is very challenging in both experiments and direct numerical simulations (DNS). In experiments of reacting flows selectively imposing conditions such as incompressibility, homogeneity or isotropy is not straightforward. Furthermore, for statistically stationary flames estimation of spectra requires two-point correlation measurements in a three-dimensional field, which are very difficult to obtain experimentally. These restrictions are alleviated somewhat in DNS, but performing well resolved DNS with a large range of dynamic scales for long durations to achieve statistical convergence is expensive, prohibitively so for reacting flows. However, with rapid increases in computing power DNS of turbulent reacting flows at realistic turbulence levels have become feasible in recent years. In the present work we employ one such DNS (Hawkes et al. 2012) to study spectra of reacting scalars in a premixed flame interacting with intense shear-driven turbulence. In terms of organisation of this paper, we first present details of the DNS in section 2. The variable density aspect of turbulent reacting flows requires a careful reconsideration of the fundamental mathematical framework required to study spectra, and this is discussed in section 3 . The results are then presented in section 4 followed by concluding remarks in section 5 .

\section{Direct numerical simulations}

Compressible three-dimensional direct numerical simulations of temporally evolving planar premixed flames interacting with a turbulent rectangular jet shear layer are considered in the present study. This configuration is amenable to analysis presented in section 3 since it results in a statistically one-dimensional turbulent premixed flame. The configuration comprises a high velocity rectangular jet of unburnt lean hydrogen-air mixture of equivalence ratio 0.7 flowing in a quiescent fluid of adiabatic burnt products of the same equivalence ratio. The unburnt reactants are preheated to $700 \mathrm{~K}$ and the pressure is $1 \mathrm{~atm}$. The jet is initialised as a high streamwise velocity region of prescribed width, $H$, with a symmetric profile about the transverse mid-plane of the computational domain. The mean streamwise velocity was prescribed to smoothly approach zero in the quiescent mixture with a hyperbolic tangent function profile given by

$$
\frac{u(y)}{U_{j}}=\frac{1}{2}\left\{\tanh \left(\frac{y / H+1 / 2}{\delta_{s 0}}\right)-\tanh \left(\frac{y / H-1 / 2}{\delta_{s 0}}\right)\right\},
$$

where $u(y)$ is the mean streamwise velocity, $U_{j}$ is the peak jet velocity, $y$ is the transverse coordinate and $\delta_{s 0}$ is the non-dimensional shear layer width which was set to 0.1. To trigger the shear layer instabilities a small amount of broadband turbulent velocity fluctuations of intensity $4 \%$ were superimposed on the initial mean velocity, and the velocity fluctuations were prescribed to approach zero in the co-flow using the same hyperbolic tangent profile as in Eq. 2.1. The initial velocity fluctuations satisfy continuity 

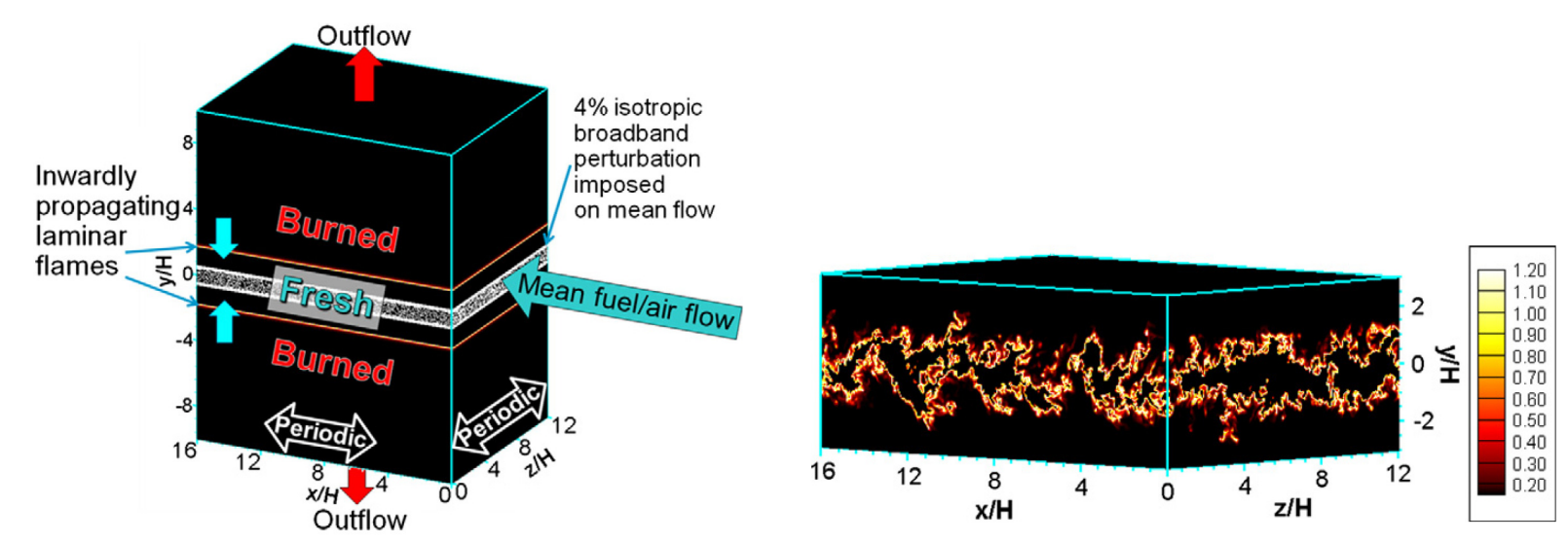

FiguRE 1. Schematic showing the configuration of the DNS (left). The streamwise direction is $x$, transverse is $y$ and spanwise is $z$. Instantaneous local heat release rate, normalised by the maximum laminar flame value, is shown on the right for the Da+ case at $t=15 t_{j}$.

and were generated using the method of Rogallo (1981) to conform to a prescribed energy spectrum of Passot \& Pouquet (1987) with an integral length scale equal to $H / 3$. Since flame interaction with a realistic turbulent shear layer was desired, two planar flames were initially placed sufficiently outside the shear layer, at symmetric locations about the transverse mid-plane, such that by the time the flames propagate inward and interact with the shear layer it would retain no memory of the initial synthetic velocity fluctuations. The planar flames divide the domain into unburnt-burnt regions with transverse profiles for temperature and species mass fractions corresponding to a freely propagating planar laminar flame. A schematic of the initialisation is given in Fig. 1 and more details are given in Hawkes et al. (2012).

The computational domain is 3D rectangular Cartesian with a size of $16 \mathrm{H} \times 20 \mathrm{H} \times 12 \mathrm{H}$ in the $x$ (streamwise), $y$ (transverse) and $z$ (spanwise) directions respectively. In the set of simulations the jet width, $H$, is varied independently while the jet velocity, $U_{j}$, is adjusted such that the jet Reynolds number, $\operatorname{Re}_{j} \equiv U_{j} H / \nu_{u}$, is held constant at 10000 while the jet Damköhler number, $\mathrm{Da}_{j} \equiv\left(H / U_{j}\right) /\left(\delta_{L} / s_{L}\right)$, varies. Here $\nu_{u}$ is the kinematic viscosity, $\delta_{L}$ is the thermal thickness and $s_{L}$ is the unstrained laminar flame speed of the unburnt mixture. Note that the definition of Damköhler number here using a mean convective time scale is slightly different from conventional definition where an integral time scale is normally used. Three simulations with Damköhler numbers $\mathrm{Da}_{j}=0.13$, 0.27 and 0.54 are performed and these will be referred to as the 'Da-', 'baseline' and 'Da+' cases respectively. The relevant numerical and thermochemical parameters are listed in Table 1. The rectangular Cartesian domain is discretised using a fixed mesh of uniform size, listed as $\Delta x$ in Table 1, in the streamwise and spanwise directions. In the transverse direction a uniform mesh of same size is used in the inner $15 \mathrm{H}$ portion of the domain, while for the outer portion the mesh is gradually coarsened since neither the flame nor the turbulent flow structures ever appear here. The grid resolution is small enough to sufficiently resolve the inner structure of the flame. The thinnest radical layer in an unstrained laminar flame for this mixture corresponds to species $\mathrm{HO}_{2}$ which has a thickness, based on full width at $90 \%$ of maximum, of $0.586 \mathrm{~mm}$, and this layer contains at least 16 grid points in the Da+ case and 32 grid points in the Da- case (see Table 1). The DNS is performed using the code S3D (Chen et al. 2009) developed at Sandia National Laboratories. S3D solves the compressible form of the conservation equations for mass, momentum, enthalpy and species mass fractions in physical space. Molecular transport 


\begin{tabular}{lccc}
\hline Parameter & Case Da- & Baseline & Case Da+ \\
\hline $\mathrm{Da}_{j}$ & 0.13 & 0.27 & 0.54 \\
$H(\mathrm{~mm})$ & 2.7 & 3.84 & 5.4 \\
$U_{j}(\mathrm{~m} / \mathrm{s})$ & 312.6 & 219.8 & 156.3 \\
$\Delta x(\mu \mathrm{m})$ & 18 & 25.6 & 36 \\
$\Delta t(\mathrm{~ns})$ & 2.5 & 4 & 5 \\
& & & \\
$s_{L}(\mathrm{~m} / \mathrm{s})$ & & 7.89 & \\
$\delta_{L}(\mathrm{~m})$ & & $5.025 \times 10^{-4}$ & \\
$\nu_{u}\left(\mathrm{~m}^{2} / \mathrm{s}\right)$ & & $8.44 \times 10^{-5}$ & \\
$\rho_{u}\left(\mathrm{~kg} / \mathrm{m}^{3}\right)$ & & 0.3961 & \\
$\rho_{b}\left(\mathrm{~kg} / \mathrm{m}^{3}\right)$ & & 0.1354 & \\
equivalence ratio & & 0.7
\end{tabular}

TABLE 1. Numerical and thermochemical parameters of the three DNS cases. The unburnt and burnt mixture densities are $\rho_{u}$ and $\rho_{b}$.

coefficients are prescribed using the mixture-averaged formulation by interfacing with the TRANSPORT library (Kee et al. 1986). Finite rate chemical kinetics are prescribed by interfacing with the CHEMKIN library (Kee et al. 1996) and the chemical mechanism of Li et al. (2004) with 9 species and 19 elementary reactions was used. S3D employs high order explicit finite difference schemes: an eighth-order central difference scheme for spatial derivatives (Kennedy \& Carpenter 1994) and a six-stage fourth-order low storage Runge-Kutta scheme for temporal derivatives (Kennedy et al. 2000). All fields of the solution vector are also filtered periodically using a tenth-order explicit filter (Kennedy \& Carpenter 1994) to remove spurious high-frequency noise. The application of the filter in physical space can have some implications for the spectral content and this is discussed in section 4. The solution is advanced using a fixed time step, $\Delta t$, given in Table 1.

Periodic boundary conditions are applied at the streamwise and spanwise boundaries. The transverse boundaries are prescribed as non-reflecting outflow using the NavierStokes characteristic boundary conditions (NSCBC) treatment of Poinsot \& Lele (1992) with the improvements of Yoo \& Im (2007). After initialisation the two flame sheets propagate inward while the turbulence in the shear layer develops. As the flame sheets interact with the shear layer they get wrinkled and corrugated causing a gradual increase in the turbulent burning velocity, defined here based on the consumption rate of hydrogen

$$
s_{T}=\frac{\int \overline{\dot{\omega}_{\mathrm{H}_{2}}} d y}{\rho_{u} \mathrm{Y}_{\mathrm{H}_{2}, \mathrm{u}}} .
$$

The overline $\overline{(. .)}$ denotes averaging in the streamwise and spanwise directions. The turbulent burning velocity rises above the laminar flame speed, $s_{L}$, and saturates before dropping to zero once all the reactants are exhausted. The variation of $s_{T} / s_{L}$ with time, normalised by the jet time $t_{j} \equiv H / U_{j}$, is shown in Fig. 2 for the Da+ and Da- cases.

\section{Mathematical framework}

\section{1. density-weighted correlation tensors}

In the study of turbulent reacting flows it is conventional to consider density-weighted moments, since their conservation equations closely resemble their incompressible counterparts and are more tractable from a modelling point of view. The mean and fluctuation 


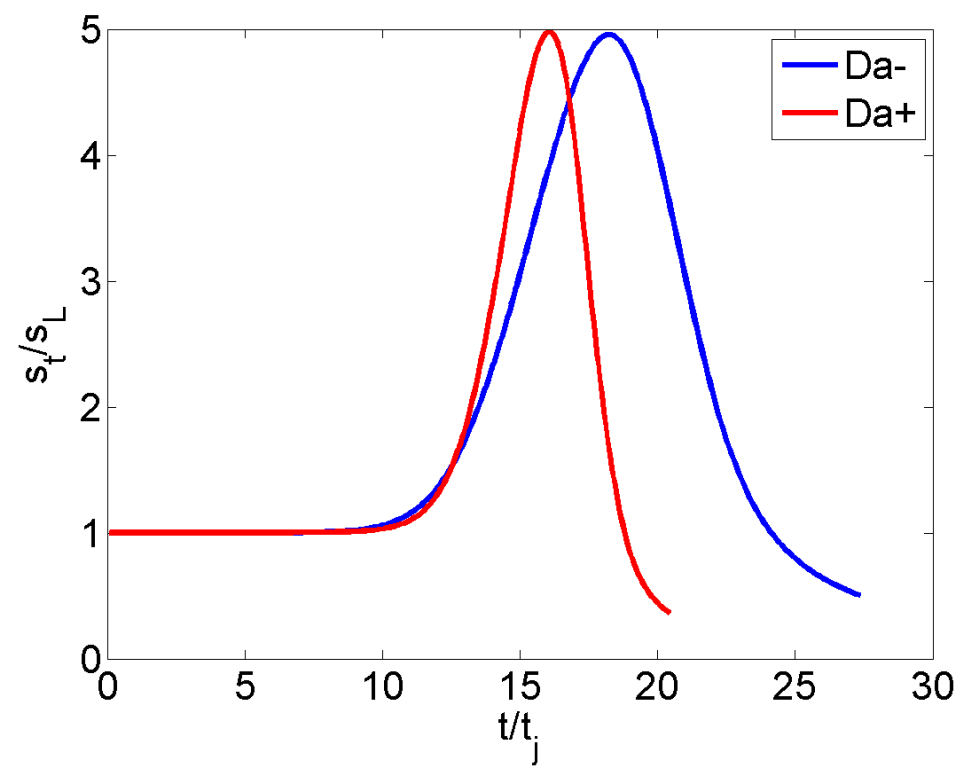

FIGURE 2. Variation of the turbulent to laminar burning velocity ratio, $s_{T} / s_{L}$, for the Da+ and Da- cases is plotted against normalised time, $t / t_{j}$.

of velocity, for instance, are defined as, respectively,:

$$
\widetilde{U}_{i}=\overline{\rho u_{i}} / \bar{\rho}, \quad u_{i}^{\prime \prime}=u_{i}-\widetilde{U}_{i}
$$

and the Reynolds-stress tensor is $\overline{\rho u_{i}^{\prime \prime} u_{j}^{\prime \prime}} / \bar{\rho}$. The same ideas are seldom extended to the study of spectral quantities leading to some potential discrepancies.

To illustrate this consider the conventional definitions of the two-point velocity crosscorrelation and velocity spectrum tensors - a Fourier transform pair - in incompressible flows (Pope 2000):

$$
\begin{aligned}
R_{i j}(\vec{r}) & =\overline{u_{i}^{\prime}(\overrightarrow{\mathrm{x}}) u_{j}^{\prime}(\overrightarrow{\mathrm{x}}+\overrightarrow{\mathrm{r}})} \\
\Phi_{i j}(\overrightarrow{\mathrm{k}}) & =\frac{1}{(2 \pi)^{3}} \iint_{-\infty}^{\infty} \exp (-i \overrightarrow{\mathrm{k}} \cdot \overrightarrow{\mathrm{r}}) R_{i j}(\overrightarrow{\mathrm{r}}) d \overrightarrow{\mathrm{r}}
\end{aligned}
$$

where $u_{i}^{\prime} \equiv u_{i}-\overline{u_{i}}$ is the velocity fluctuation about the conventional ensemble average, $\overrightarrow{\mathrm{x}}, \overrightarrow{\mathrm{r}}$ are position and displacement vectors in physical space, respectively, and $\overrightarrow{\mathrm{k}}$ is the wavenumber vector. Thus defined, the turbulent kinetic energy, t.k.e., is related to the velocity spectrum tensor via

$$
\text { t.k.e. }=\frac{1}{2} \overline{u_{i}^{\prime} u_{i}^{\prime}}=\frac{1}{2} \iint_{-\infty}^{\infty} \int_{i i}(\overrightarrow{\mathrm{k}}) d \overrightarrow{\mathrm{k}} .
$$

An energy spectrum function, $E(k)$, can then be defined which represents the contribution to $t$.k.e. from all wavenumbers of magnitude $k$ (i.e. $k=|\overrightarrow{\mathrm{k}}|$ ) such that

$$
E(k)=\frac{1}{2} \iint_{-\infty}^{\infty} \int_{i i}(\overrightarrow{\mathrm{k}}) \delta(|\overrightarrow{\mathrm{k}}|-k) d \overrightarrow{\mathrm{k}}, \text { and t.k.e. }=\int_{0}^{\infty} E(k) d k .
$$


In a discrete representation of the flow field, such as in a DNS, $E(k)$ can be constructed as the average of the product of all the Fourier coefficients of $u_{i}^{\prime}$ with a wavenumber magnitude of $k$.

If one were to naïvely replace $u_{i}^{\prime}$ with $u_{i}^{\prime \prime}$ in the above schema one would find that the resulting energy spectrum function recovers $\overline{u_{i}^{\prime \prime} u_{i}^{\prime \prime}} / 2$ which is not equal to the densityweighted turbulent kinetic energy, $\widetilde{t . k . e} . \equiv \overline{\rho u_{i}^{\prime \prime} u_{i}^{\prime \prime}} / 2 \bar{\rho}$. In other words the Fourier coefficients of $u_{i}^{\prime \prime}$ cannot be used to represent the spectrum function of $\widetilde{t . k . e .}$. Considering the almost universal adoption of density-weighted quantities in the study and modelling of turbulent reacting flows, an appropriate mathematical framework is in order. To this end, we propose that the two-point velocity cross-correlation tensor incorporate a density weighting as follows:

$$
\widetilde{R}_{i j}(\overrightarrow{\mathrm{r}})=\frac{1}{2 \bar{\rho}}\left[\overline{\rho(\overrightarrow{\mathrm{x}}) u_{i}^{\prime \prime}(\overrightarrow{\mathrm{x}}) u_{j}^{\prime \prime}(\overrightarrow{\mathrm{x}}+\overrightarrow{\mathrm{r}})}+\overline{u_{i}^{\prime \prime}(\overrightarrow{\mathrm{x}}) \rho(\overrightarrow{\mathrm{x}}+\overrightarrow{\mathrm{r}}) u_{j}^{\prime \prime}(\overrightarrow{\mathrm{x}}+\overrightarrow{\mathrm{r}})}\right] .
$$

Strictly, a trivariate correlation tensor should involve two separation vectors and in that respect the tensor defined above represents a contraction along one vector. The reasons for this choice are twofold. First, in variable density flows momentum, rather than velocity is the fundamentally conserved quantity. Physically, $\widetilde{R}_{i j}$ can be interpreted as the influence, on average, of the momentum fluctuation at point $(\vec{x})$ on the velocity fluctuation at point $(\vec{x}+\vec{r})$, and vice-versa. Second, as the ensuing derivations will show, this contraction simplifies the derivation of a balance equation for $\widetilde{R}_{i j}$ with each of the terms carrying a clear physical meaning. Yoshizawa et al. (2013) found a similar benefit, simplified balance equations for single-point statistical moments, by weighting the fluctuating quantities by density. It is evident that $\widetilde{R}_{i j}(0)$ is a symmetric tensor $\left(\widetilde{R}_{i j}(0)=\widetilde{R}_{j i}(0)\right)$ and it is equal to the density-weighted Reynolds-stress tensor. If the density and fluctuating velocity fields are homogeneous then $\widetilde{R}_{i j}(\overrightarrow{\mathrm{r}})=\widetilde{R}_{j i}(-\overrightarrow{\mathrm{r}})$, a property shared by its incompressible counterpart $R_{i j}$. Homogeneity might seem a stringent assumption for reacting flows but one might conceive of statistically one-dimensional reacting flows with a fixed mean flame normal direction such that homogeneity applies in the two directions that are orthogonal to it. However, while the continuity equation in incompressible flows yields the simplifications

$$
\begin{aligned}
\nabla \cdot \mathrm{u}^{\prime} & =0, \\
\frac{\partial R_{i j}}{\partial r_{j}} & =0,
\end{aligned}
$$

the same for $\widetilde{R}_{i j}$ is not afforded by the definition in Eq. 3.5. Furthermore, for incompressible isotropic turbulence there exist simple relationships between the two-point correlation tensors and velocity structure functions which also contain scale information. This is clealry not the case for the density weighted correlation tensor. One could contrive density weighted structure functions to be consistent with the correlation tensors but that is not an objective here. Nonetheless, analogous definitions for density-weighted 
velocity spectrum tensor and energy spectrum function can be written:

$$
\begin{aligned}
\widetilde{\Phi}_{i j}(\overrightarrow{\mathrm{k}}) & =\frac{1}{(2 \pi)^{3}} \iint_{-\infty}^{\infty} \exp (-i \overrightarrow{\mathrm{k}} \cdot \overrightarrow{\mathrm{r}}) \widetilde{R}_{i j}(\overrightarrow{\mathrm{r}}) d \overrightarrow{\mathrm{r}}, \\
\widetilde{E}(k) & =\frac{1}{2} \iint_{-\infty}^{\infty} \int_{\Phi_{i i}(\overrightarrow{\mathrm{k}}) \delta(|\overrightarrow{\mathrm{k}}|-k) d \overrightarrow{\mathrm{k}},} \\
\widetilde{\text { t.k.e. }} & =\int_{0}^{\infty} \widetilde{E}(k) d k .
\end{aligned}
$$

Likewise, the density-weighted cross-correlation tensor between two fluctuating scalars $\psi$ and $\xi$ can be defined as

$$
\widetilde{R}_{\psi \xi}(\overrightarrow{\mathrm{r}})=\frac{1}{2 \bar{\rho}}\left[\overline{\rho(\overrightarrow{\mathrm{x}}) \psi^{\prime \prime}(\overrightarrow{\mathrm{x}}) \xi^{\prime \prime}(\overrightarrow{\mathrm{x}}+\overrightarrow{\mathrm{r}})}+\overline{\psi^{\prime \prime}(\overrightarrow{\mathrm{x}}) \rho(\overrightarrow{\mathrm{x}}+\overrightarrow{\mathrm{r}}) \xi^{\prime \prime}(\overrightarrow{\mathrm{x}}+\overrightarrow{\mathrm{r}})}\right] .
$$

The important distinction that must be stressed here is that the spectrum function for kinetic energy, $\widetilde{E}(k)$, must now be constructed as the average of the product of the Fourier coefficients of $\rho u_{i}^{\prime \prime}$ and $u_{i}^{\prime \prime}$. Note that this represents a one-wavenumber spectral decomposition of the Favre averaged turbulent kinetic energy and it stems from the choice of using only one spearation vector in the definition of $\widetilde{R}_{i j}$. Similarly, for scalar

energy, $\widetilde{E}_{\psi}(k)$, it must be the average of the product of Fourier coefficients of $\rho \psi \prime \prime$ and $\psi \prime \prime$.

The density weighting for the correlation tensors proposed here is not a definitive form for variable density flows and other forms are certainly possible that might retain all the properties of the incompressible counterparts. The proposed definitions are motivated by the need to capture scale information while still being consistent with Favre-averaged Reynolds stresses and scalar fluctuations which are widely considered as useful quantities for describing turbulent reacting flows. Apart from having the useful properties described above, these definitions of $\widetilde{R}_{i j}$ and $\widetilde{R}_{\psi \xi}$ serve an important objective of the present study; the derivation of a balance equation for the spectrum of turbulent kinetic energy and scalar fluctuations in reacting flows, presented in the following sub-section.

\subsection{Balance equations for density-weighted spectrum functions}

Hinze (1975) presents an elegant derivation of a balance equation for two-point velocity correlations, leading to an equation for the energy spectrum function, in incompressible isotropic turbulence as well as in homogeneous sheared turbulence. He also notes the difficulties of incorporating variable density, particularly if the local turbulent fluctuations are high enough that compressibility effects become appreciable. Krzywoblocki (1952) derived balance equations for velocity and temperature fluctuation correlations in compressible homogeneous isotropic turbulence but considered a density-weighted correlation function of the form $\overline{\rho(\overrightarrow{\mathrm{x}}) u_{i}^{\prime \prime}(\overrightarrow{\mathrm{x}}) \rho(\overrightarrow{\mathrm{x}}+\overrightarrow{\mathrm{r}}) u_{j}^{\prime \prime}(\overrightarrow{\mathrm{x}}+\overrightarrow{\mathrm{r}})}$, which is different from Eq. 3.5. Here we seek a balance equation governing the latter.

In the derivation to follow we largely adopt the approach and notation of Hinze (1975). For the sake of simplicity, and without loss of generality, we consider statistically stationary turbulent reacting flows. Furthermore, since reacting flows can not be homogeneous in a strict three-dimensional sense, consider a scenario that comes closest, i.e. statistically one-dimensional reacting flows, where moments vary only along a mean flame normal direction. The two-point correlation function $\widetilde{R}_{i j}(\overrightarrow{\mathrm{r}})$ will then be invariant to translation 
as long as the displacement vector $\overrightarrow{\mathrm{r}}$ is constrained to always be orthogonal to the mean flame normal vector. We start from the balance equations derived in appendix $\mathrm{A}$ for $u_{i}^{\prime \prime}$ (Eq. A 4),

$$
\frac{\partial u_{i}^{\prime \prime}}{\partial t}+\frac{\partial \widetilde{U}_{i}}{\partial t}+\widetilde{U}_{k} \frac{\partial \widetilde{U}_{i}}{\partial x_{k}}+u_{k}^{\prime \prime} \frac{\partial \widetilde{U}_{i}}{\partial x_{k}}+\widetilde{U}_{k} \frac{\partial u_{i}^{\prime \prime}}{\partial x_{k}}+u_{k}^{\prime \prime} \frac{\partial u_{i}^{\prime \prime}}{\partial x_{k}}=-\frac{1}{\rho} \frac{\partial\left(\bar{P}+p^{\prime}\right)}{\partial x_{i}}+\frac{1}{\rho} \frac{\partial \tau_{i k}}{\partial x_{k}},
$$

and for $\rho u_{i}^{\prime \prime}$ (Eq. A 8),

$\frac{\partial \rho u_{i}^{\prime \prime}}{\partial t}+\rho^{\prime}\left(\frac{\partial \widetilde{U}_{i}}{\partial t}+\widetilde{U}_{k} \frac{\partial \widetilde{U}_{i}}{\partial x_{k}}\right)+\rho u_{k}^{\prime \prime} \frac{\partial \widetilde{U}_{i}}{\partial x_{k}}+\frac{\partial \rho u_{i}^{\prime \prime} \widetilde{U}_{k}}{\partial x_{k}}+\frac{\partial}{\partial x_{k}}\left(\rho u_{i}^{\prime \prime} u_{k}^{\prime \prime}-\overline{\rho u_{i}^{\prime \prime} u_{k}^{\prime \prime}}\right)=-\frac{\partial p^{\prime}}{\partial x_{i}}+\frac{\partial \tau_{i k}^{\prime}}{\partial x_{k}}$.

Denoting locations $\overrightarrow{\mathrm{x}}$ and $(\overrightarrow{\mathrm{x}}+\overrightarrow{\mathrm{r}})$ by ()$_{A}$ and ()$_{B}$, respectively, the equation for $\left(u_{i}^{\prime \prime}\right)_{A}$ is multiplied by $\left(\rho u_{j}^{\prime \prime}\right)_{B}$ and equation for $\left(\rho u_{i}^{\prime \prime}\right)_{A}$ is multiplied by $\left(u_{j}^{\prime \prime}\right)_{B}$. Similarly the equations for $\left(u_{j}^{\prime \prime}\right)_{B}$ and $\left(\rho u_{j}^{\prime \prime}\right)_{B}$ are multiplied by $\left(\rho u_{i}^{\prime \prime}\right)_{A}$ and $\left(u_{i}^{\prime \prime}\right)_{A}$, respectively, and the resulting four equations are added. Note that the derivative at point $\mathrm{A}$ of any quantity at point $\mathrm{B}$ is zero, and vice-versa. Averaging the result, noting that $\overline{\rho u^{\prime \prime}}=0$, gives

$$
\begin{aligned}
& \frac{2 \partial\left(\bar{\rho} \widetilde{R}_{i j}\right)}{\partial t}+\overline{\rho^{\prime}{ }_{A}\left(u_{j}^{\prime \prime}\right)_{B}}\left(\frac{\partial \widetilde{U}_{i}}{\partial t}+\widetilde{U}_{k} \frac{\partial \widetilde{U}_{i}}{\partial x_{k}}\right)_{A}+\overline{\rho_{B}^{\prime}\left(u_{i}^{\prime \prime}\right)_{A}}\left(\frac{\partial \widetilde{U}_{j}}{\partial t}+\widetilde{U}_{k} \frac{\partial \widetilde{U}_{j}}{\partial x_{k}}\right)_{B} \\
& +\overline{\left(u_{j}^{\prime \prime}\right)_{B}\left(\rho u_{k}^{\prime \prime}\right)_{A}}\left(\frac{\partial \widetilde{U}_{i}}{\partial x_{k}}\right)_{A}+\overline{\left(\rho u_{j}^{\prime \prime}\right)_{B}\left(u_{k}^{\prime \prime}\right)_{A}}\left(\frac{\partial \widetilde{U}_{i}}{\partial x_{k}}\right)_{A}+\overline{\left(u_{i}^{\prime \prime}\right)_{A}\left(\rho u_{k}^{\prime \prime}\right)_{B}}\left(\frac{\partial \widetilde{U}_{j}}{\partial x_{k}}\right)_{B}+\overline{\left(\rho u_{i}^{\prime \prime}\right)_{A}\left(u_{k}^{\prime \prime}\right)_{B}}\left(\frac{\partial \widetilde{U}_{j}}{\partial x_{k}}\right)_{B} \\
& +\left(\frac{\partial}{\partial x_{k}}\right)_{A}\left(\widetilde{U}_{k}\right)_{A} \overline{\left(\rho u_{i}^{\prime \prime}\right)_{A}\left(u_{j}^{\prime \prime}\right)_{B}}+\left(\widetilde{U}_{k} \frac{\partial}{\partial x_{k}}\right)_{A} \overline{\left(u_{i}^{\prime \prime}\right)_{A}\left(\rho u_{j}^{\prime \prime}\right)_{B}}+\left(\frac{\partial}{\partial x_{k}}\right)_{B}\left(\widetilde{U}_{k}\right)_{B} \overline{\left(\rho u_{j}^{\prime \prime}\right)_{B}\left(u_{i}^{\prime \prime}\right)_{A}}+\left(\widetilde{U}_{k} \frac{\partial}{\partial x_{k}}\right)_{B} \overline{\left(u_{j}^{\prime \prime}\right)_{B}\left(\rho u_{i}^{\prime \prime}\right)_{A}} \\
& +\left(\frac{\partial}{\partial x_{k}}\right)_{A} \overline{\left(\rho u_{i}^{\prime \prime} u_{k}^{\prime \prime}\right)_{A}\left(u_{j}^{\prime \prime}\right)_{B}}+\overline{\left(\rho u_{j}^{\prime \prime}\right)_{B}\left(u_{k}^{\prime \prime} \frac{\partial u_{i}^{\prime \prime}}{\partial x_{k}}\right)_{A}}+\left(\frac{\partial}{\partial x_{k}}\right)_{B} \overline{\left(\rho u_{j}^{\prime \prime} u_{k}^{\prime \prime}\right)_{B}\left(u_{i}^{\prime \prime}\right)_{A}}+\overline{\left(\rho u_{i}^{\prime \prime}\right)_{A}\left(u_{k}^{\prime \prime} \frac{\partial u_{j}^{\prime \prime}}{\partial x_{k}}\right)_{B}} \\
& -\overline{\left(u_{i}^{\prime \prime}\right)_{A}}\left(\frac{\partial \overline{\rho u_{j}^{\prime \prime} u_{k}^{\prime \prime}}}{\partial x_{k}}\right)_{B}-\overline{\left(u_{j}^{\prime \prime}\right)_{B}}\left(\frac{\partial \overline{\rho u_{i}^{\prime \prime} u_{k}^{\prime \prime}}}{\partial x_{k}}\right)_{A}= \\
& -\left(\frac{\partial}{\partial x_{i}}\right)_{A} \overline{p_{A}^{\prime}\left(u_{j}^{\prime \prime}\right)_{B}}-\left(\frac{\partial}{\partial x_{j}}\right)_{B} \overline{p_{B}^{\prime}\left(u_{i}^{\prime \prime}\right)_{A}}-\overline{\left(\rho u_{i}^{\prime \prime}\right)_{A}\left(\frac{1}{\rho} \frac{\partial\left(p^{\prime}+\bar{P}\right)}{\partial x_{j}}\right)_{B}}-\overline{\left(\rho u_{j}^{\prime \prime}\right)_{B}\left(\frac{1}{\rho} \frac{\partial\left(p^{\prime}+\bar{P}\right)}{\partial x_{i}}\right)_{A}} \\
& +\left(\frac{\partial}{\partial x_{k}}\right)_{A} \overline{\left(\tau_{i k}^{\prime}\right)_{A}\left(u_{j}^{\prime \prime}\right)_{B}}+\left(\frac{\partial}{\partial x_{k}}\right)_{B} \overline{\left(\tau_{j k}^{\prime}\right)_{B}\left(u_{i}^{\prime \prime}\right)_{A}}+\overline{\left(\rho u_{i}^{\prime \prime}\right)_{A}\left(\frac{1}{\rho} \frac{\partial \tau_{j k}}{\partial x_{k}}\right)_{B}}+\overline{\left(\rho u_{j}^{\prime \prime}\right)_{B}\left(\frac{1}{\rho} \frac{\partial \tau_{i k}}{\partial x_{k}}\right)_{A}} .
\end{aligned}
$$

A few simplifications are in order here. The fourth and fifth terms on the left hand side of Eq. 3.11 are simply $2 \bar{\rho} \widetilde{R}_{k j}\left(\partial \widetilde{U}_{i} / \partial x_{k}\right)_{A}$, while the sixth and seventh terms are $2 \bar{\rho} \widetilde{R}_{i k}\left(\partial \widetilde{U}_{j} / \partial x_{k}\right)_{B}$. The second and sixteenth terms can be combined and rewritten as follows:

$$
\begin{gathered}
\overline{\left(\rho_{A}^{\prime}-\rho_{B}^{\prime}\right)\left(u_{j}^{\prime \prime}\right)_{B}}\left(\frac{\partial \widetilde{U}_{i}}{\partial t}+\widetilde{U}_{k} \frac{\partial \widetilde{U}_{i}}{\partial x_{k}}\right)_{A}+\overline{\rho_{B}^{\prime}{ }_{B}\left(u_{j}^{\prime \prime}\right)_{B}}\left(\frac{\partial \widetilde{U}_{i}}{\partial t}+\widetilde{U}_{k} \frac{\partial \widetilde{U}_{i}}{\partial x_{k}}\right)_{A}-\overline{\left(u_{j}^{\prime \prime}\right)_{B}}\left(\frac{\partial \overline{\rho u_{i}^{\prime \prime} u_{k}^{\prime \prime}}}{\partial x_{k}}\right)_{A} \\
=\overline{\left(\rho_{A}^{\prime}-\rho_{B}^{\prime}\right)\left(u_{j}^{\prime \prime}\right)_{B}}\left(\frac{\partial \widetilde{U}_{i}}{\partial t}+\widetilde{U}_{k} \frac{\partial \widetilde{U}_{i}}{\partial x_{k}}\right)_{A}-\overline{\left(u_{j}^{\prime \prime}\right)_{B}}\left(\bar{\rho} \frac{\partial \widetilde{U}_{i}}{\partial t}+\bar{\rho} \widetilde{U}_{k} \frac{\partial \widetilde{U}_{i}}{\partial x_{k}}+\frac{\partial \overline{\rho u_{i}^{\prime \prime} u_{k}^{\prime \prime}}}{\partial x_{k}}\right)_{A} \\
=\overline{\left(\rho_{A}^{\prime}-\rho_{B}^{\prime}\right)\left(u_{j}^{\prime \prime}\right)_{B}}\left(\frac{\partial \widetilde{U}_{i}}{\partial t}+\widetilde{U}_{k} \frac{\partial \widetilde{U}_{i}}{\partial x_{k}}\right)_{A}-\overline{\left(u_{j}^{\prime \prime}\right)_{B}}\left(-\frac{\partial \bar{P}}{\partial x_{i}}+\frac{\partial \bar{\tau}_{i k}}{\partial x_{k}}\right)_{A}
\end{gathered}
$$


after using Eqs. A 6 and A 7 for a stationary flow. Similarly, the third and seventeenth terms can be rewritten as

$$
\begin{gathered}
\overline{\left(\rho_{B}^{\prime}-\rho_{A}^{\prime}\right)\left(u_{i}^{\prime \prime}\right)_{A}}\left(\frac{\partial \widetilde{U}_{j}}{\partial t}+\widetilde{U}_{k} \frac{\partial \widetilde{U}_{j}}{\partial x_{k}}\right)_{B}+\overline{\rho^{\prime}{ }_{A}\left(u_{i}^{\prime \prime}\right)_{A}}\left(\frac{\partial \widetilde{U}_{j}}{\partial t}+\widetilde{U}_{k} \frac{\partial \widetilde{U}_{j}}{\partial x_{k}}\right)_{B}-\overline{\left(u_{i}^{\prime \prime}\right)_{A}}\left(\frac{\partial \overline{\rho u_{j}^{\prime \prime} u_{k}^{\prime \prime}}}{\partial x_{k}}\right)_{B} \\
=\overline{\left(\rho_{B}^{\prime}-\rho_{A}^{\prime}\right)\left(u_{i}^{\prime \prime}\right)_{A}}\left(\frac{\partial \widetilde{U}_{j}}{\partial t}+\widetilde{U}_{k} \frac{\partial \widetilde{U}_{j}}{\partial x_{k}}\right)_{B}-\overline{\left(u_{i}^{\prime \prime}\right)_{A}}\left(-\frac{\partial \bar{P}}{\partial x_{j}}+\frac{\partial \bar{\tau}_{j k}}{\partial x_{k}}\right)_{B} .
\end{gathered}
$$

In making these simplifications it is assumed that $\overline{(\rho)_{A}}=\overline{(\rho)_{B}}$ and $\left(\partial \widetilde{U}_{k} / \partial x_{k}\right)_{A}=$ $\left(\partial \widetilde{U}_{k} / \partial x_{k}\right)_{B}$ which is valid for the statistically one-dimensional case considered here. For low Mach number reactive flows compressibility effects will be negligible and the density change occurs chiefly via chemical reactions. Accordingly, the mean density will vary only along the mean flame normal direction. In addition, following Hinze (1975), independent variables are introduced to simplify the derivatives of the two-point correlations:

$$
r_{k}=\left(x_{k}\right)_{B}-\left(x_{k}\right)_{A} ; \quad\left(x_{k}\right)_{A B}=\left[\left(x_{k}\right)_{A}+\left(x_{k}\right)_{B}\right] / 2,
$$

such that the derivatives become

$$
\begin{aligned}
& \left(\frac{\partial}{\partial x_{k}}\right)_{A}=\frac{1}{2}\left(\frac{\partial}{\partial x_{k}}\right)_{A B}-\frac{\partial}{\partial r_{k}} \\
& \left(\frac{\partial}{\partial x_{k}}\right)_{B}=\frac{1}{2}\left(\frac{\partial}{\partial x_{k}}\right)_{A B}+\frac{\partial}{\partial r_{k}} .
\end{aligned}
$$

For the statistically one-dimensional case, since the displacement vector $\vec{r}$ is constrained such that all two-point correlations are invariant to translations, derivatives of all correlations with respect to $\left(x_{k}\right)_{A B}$ are identically zero. This allows the simplification of eighth and eleventh terms on the left hand side to

$$
\begin{aligned}
\left(\frac{\partial}{\partial x_{k}}\right)_{A}\left(\widetilde{U}_{k}\right)_{A} \overline{\left(\rho u_{i}^{\prime \prime}\right)_{A}\left(u_{j}^{\prime \prime}\right)_{B}} & +\left(\widetilde{U}_{k} \frac{\partial}{\partial x_{k}}\right)_{B} \overline{\left(u_{j}^{\prime \prime}\right)_{B}\left(\rho u_{i}^{\prime \prime}\right)_{A}} \\
=-\widetilde{U}_{k} \frac{\partial}{\partial r_{k}} \overline{\left(\rho u_{i}^{\prime \prime}\right)_{A}\left(u_{j}^{\prime \prime}\right)_{B}}-\overline{\left(\rho u_{i}^{\prime \prime}\right)_{A}\left(u_{j}^{\prime \prime}\right)_{B}} \frac{\partial \widetilde{U}_{k}}{\partial r_{k}} & +\widetilde{U}_{k} \frac{\partial}{\partial r_{k}} \overline{\left(\rho u_{i}^{\prime \prime}\right)_{A}\left(u_{j}^{\prime \prime}\right)_{B}} \\
& =-\overline{\left(\rho u_{i}^{\prime \prime}\right)_{A}\left(u_{j}^{\prime \prime}\right)_{B}} \frac{\partial \widetilde{U}_{k}}{\partial r_{k}},
\end{aligned}
$$

and, similarly, the ninth and tenth terms to

$$
\left(\widetilde{U}_{k} \frac{\partial}{\partial x_{k}}\right)_{A} \overline{\left(u_{i}^{\prime \prime}\right)_{A}\left(\rho u_{j}^{\prime \prime}\right)_{B}}+\left(\frac{\partial}{\partial x_{k}}\right)_{B}\left(\widetilde{U}_{k}\right)_{B} \overline{\left(u_{i}^{\prime \prime}\right)_{A}\left(\rho u_{j}^{\prime \prime}\right)_{B}}=\overline{\left(u_{i}^{\prime \prime}\right)_{A}\left(\rho u_{j}^{\prime \prime}\right)_{B}} \frac{\partial \widetilde{U}_{k}}{\partial r_{k}} .
$$

Finally, the twelfth and thirteenth terms are simplified to

$$
\begin{aligned}
\left(\frac{\partial}{\partial x_{k}}\right)_{A} \overline{\left(\rho u_{i}^{\prime \prime} u_{k}^{\prime \prime}\right)_{A}\left(u_{j}^{\prime \prime}\right)_{B}}+\overline{\left(\rho u_{j}^{\prime \prime}\right)_{B}\left(u_{k}^{\prime \prime} \frac{\partial u_{i}^{\prime \prime}}{\partial x_{k}}\right)_{A}} \\
=\left(\frac{\partial}{\partial x_{k}}\right)_{A} \overline{\left(\rho u_{i}^{\prime \prime} u_{k}^{\prime \prime}\right)_{A}\left(u_{j}^{\prime \prime}\right)_{B}}+\overline{\left(\rho u_{j}^{\prime \prime}\right)_{B}\left[\frac{\partial u_{i}^{\prime \prime} u_{k}^{\prime \prime}}{\partial x_{k}}-u_{i}^{\prime \prime} \frac{\partial u_{k}^{\prime \prime}}{\partial x_{k}}\right]_{A}} \\
=\left(\frac{\partial}{\partial x_{k}}\right)_{A}\left[\overline{\left(\rho u_{i}^{\prime \prime} u_{k}^{\prime \prime}\right)_{A}\left(u_{j}^{\prime \prime}\right)_{B}}+\overline{\left(u_{i}^{\prime \prime} u_{k}^{\prime \prime}\right)_{A}\left(\rho u_{j}^{\prime \prime}\right)_{B}}\right]-\overline{\left(\rho u_{j}^{\prime \prime}\right)_{B}\left(u_{i}^{\prime \prime} \frac{\partial u_{k}^{\prime \prime}}{\partial x_{k}}\right)_{A}}
\end{aligned}
$$


while the fourteenth and fifteenth terms to

$$
\left(\frac{\partial}{\partial x_{k}}\right)_{B}\left[\overline{\left(\rho u_{j}^{\prime \prime} u_{k}^{\prime \prime}\right)_{B}\left(u_{i}^{\prime \prime}\right)_{A}}+\overline{\left(u_{j}^{\prime \prime} u_{k}^{\prime \prime}\right)_{B}\left(\rho u_{i}^{\prime \prime}\right)_{A}}\right]-\overline{\left(\rho u_{i}^{\prime \prime}\right)_{A}\left(u_{j}^{\prime \prime} \frac{\partial u_{k}^{\prime \prime}}{\partial x_{k}}\right)_{B}} .
$$
$\widetilde{R}_{i j}$ :

$$
\begin{aligned}
& \frac{2 \partial \bar{\rho} \widetilde{R}_{i j}}{\partial t}+\underbrace{2 \bar{\rho} \widetilde{R}_{i k}\left(\frac{\partial \widetilde{U}_{j}}{\partial r_{k}}\right)-2 \bar{\rho} \widetilde{R}_{k j}\left(\frac{\partial \widetilde{U}_{i}}{\partial r_{k}}\right)}_{\mathrm{T} 1}+\underbrace{\left[\overline{\left(u_{i}^{\prime \prime}\right)_{A}\left(\rho u_{j}^{\prime \prime}\right)_{B}}-\overline{\left(\rho u_{i}^{\prime \prime}\right)_{A}\left(u_{j}^{\prime \prime}\right)_{B}}\right]\left(\frac{\partial \widetilde{U}_{k}}{\partial r_{k}}\right)}_{\mathrm{T} 2} \\
& -\underbrace{\left(\frac{\partial}{\partial r_{k}}\right)\left[\overline{\left(\rho u_{i}^{\prime \prime} u_{k}^{\prime \prime}\right)_{A}\left(u_{j}^{\prime \prime}\right)_{B}}+\overline{\left(u_{i}^{\prime \prime} u_{k}^{\prime \prime}\right)_{A}\left(\rho u_{j}^{\prime \prime}\right)_{B}}\right]+\left(\frac{\partial}{\partial r_{k}}\right)\left[\overline{\left(u_{i}^{\prime \prime}\right)_{A}\left(\rho u_{j}^{\prime \prime} u_{k}^{\prime \prime}\right)_{B}}+\overline{\left(\rho u_{i}^{\prime \prime}\right)_{A}\left(u_{j}^{\prime \prime} u_{k}^{\prime \prime}\right)_{B}}\right]}_{\text {T3 } 3_{1}}
\end{aligned}
$$

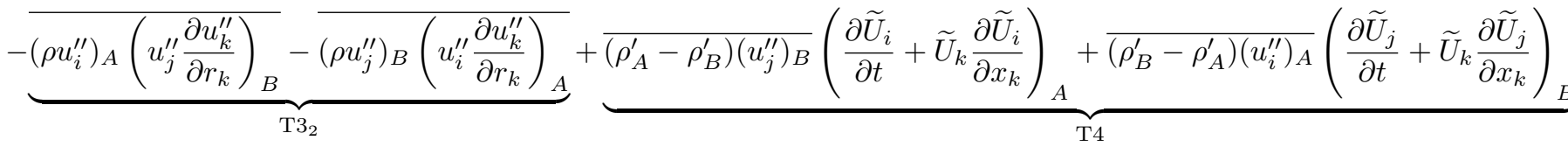

$$
\begin{aligned}
& =+\underbrace{\left(\frac{\partial}{\partial r_{i}}\right) \overline{\left(p^{\prime}+\bar{P}\right)_{A}\left(u_{j}^{\prime \prime}\right)_{B}}-\left(\frac{\partial}{\partial r_{j}}\right) \overline{\left(p^{\prime}+\bar{P}\right)_{B}\left(u_{i}^{\prime \prime}\right)_{A}}}_{\mathrm{T} 5_{1}}-\underbrace{\left(\rho u_{i}^{\prime \prime}\right)_{A}\left(\frac{1}{\rho} \frac{\partial\left(p^{\prime}+\bar{P}\right)}{\partial x_{j}}\right)_{B}}_{\mathrm{T} 5_{2}}-\overline{\left.u_{j}^{\prime \prime}\right)_{B}\left(\frac{1}{\rho} \frac{\partial\left(p^{\prime}+\bar{P}\right)}{\partial x_{i}}\right)_{A}} \\
& -\underbrace{\left(\frac{\partial}{\partial r_{k}}\right) \overline{\left(\tau_{i k}\right)_{A}\left(u_{j}^{\prime \prime}\right)_{B}}+\left(\frac{\partial}{\partial r_{k}}\right) \overline{\left(\tau_{j k}\right)_{B}\left(u_{i}^{\prime \prime}\right)_{A}}}_{\mathrm{T} 6_{1}}+\underbrace{\overline{\left(\rho u_{i}^{\prime \prime}\right)_{A}\left(\frac{1}{\rho} \frac{\partial \tau_{j k}}{\partial x_{k}}\right)_{B}}+\overline{\left(\rho u_{j}^{\prime \prime}\right)_{B}\left(\frac{1}{\rho} \frac{\partial \tau_{i k}}{\partial x_{k}}\right)_{A}}}_{\mathrm{T} 6_{2}} .
\end{aligned}
$$

The physical interpretation of the various terms is as follows: T1 represents the contribution of mean velocity gradients; T2 represents the interaction between the mean velocity and the fluctuating velocity correlations and can be interpreted as eddy deformation by mean flow (Hinze 1975); $\mathrm{T} 3 \equiv\left(\mathrm{T} 3_{1}+\mathrm{T} 3_{2}\right)$ denotes the contribution of the turbulent straining motions; T4 represents the influence purely due to density fluctuations; $\mathrm{T} 5 \equiv\left(\mathrm{T} 5_{1}+\mathrm{T} 5_{2}\right)$ represents the contribution of pressure-velocity correlations and $\mathrm{T} 6 \equiv\left(\mathrm{T} 6_{1}+\mathrm{T} 6_{2}\right)$ represents the viscous dissipation effects. All of the terms in Eq. 3.12, except the fluctuating density term T4, have analogues in the corresponding equation presented by Hinze (1975) for the incompressible case. Without the density weighting ascribed to the definition of $\widetilde{R}_{i j}$, arriving at an equation with such clear physical meaning of the various terms would not have been possible. If one were to instead attempt a derivation without the density weighting, the resulting equation would be much more complicated and unwieldy.

Our objective here is not to attempt a closure for the various terms in Eq. 3.12, but rather to illustrate the various physical processes in reacting flows and compare them with the incompressible case. We do not present any further simplification of this equation but rather intend to use it as a basis to study the energy balance in wavenumber space. Hinze (1975) considers the special case of homogeneous isotropic sheared turbulence with a non-zero but uniform mean velocity gradient which simplifies the equation considerably, particularly the terms involving mean velocity and its gradients. Such an exercise is of limited utility here. The simplest archetypes of statistically one-dimensional reacting flows conceivable - a non-premixed flame in a turbulent shear layer or a statistically 
planar freely propagating premixed flame - do not satisfy the condition of uniform mean velocity gradient.

We now apply the Fourier transform operation, denoted by the operator

$$
\mathcal{F}\{. .\}=\frac{1}{(2 \pi)^{3}} \iint_{-\infty}^{\infty} \exp (-i \overrightarrow{\mathrm{k}} \cdot \overrightarrow{\mathrm{r}})(. .) d \overrightarrow{\mathrm{r}},
$$

to each of the terms in Eq. 3.12 and contract the tensorial indices $i$ and $j$. The result is expressed nominally as

$$
2 \bar{\rho} \frac{\partial \widetilde{\Phi}_{i i}(\overrightarrow{\mathrm{k}})}{\partial t}+\mathcal{F}\{\mathrm{T} 1\}+\mathcal{F}\{\mathrm{T} 2\}+\mathcal{F}\{\mathrm{T} 3\}+\mathcal{F}\{\mathrm{T} 4\}=\mathcal{F}\{\mathrm{T} 5\}+\mathcal{F}\{\mathrm{T} 6\} .
$$

Since $\widetilde{\Phi}_{i i}(\overrightarrow{\mathrm{k}})$ represents the contribution to $\widetilde{t . k . e}$. from the wavenumber $\overrightarrow{\mathrm{k}}$, Eq. 3.13 denotes the balance of $\widetilde{t . k . e}$. in wavenumber space. Upon closer inspection of each of the terms it is self evident that the terms $\mathcal{F}\{\mathrm{T} 1\}$ and $\mathcal{F}\{\mathrm{T} 2\}$ represent energy production due to large-scale mean motion, just as their incompressible counterparts. On the other hand the term $\mathcal{F}\{\mathrm{T} 4\}$ denotes an additional mechanism by which mean motion contributes to energy production by coupling with fluctuating density-velocity correlations, a mechanism absent in the incompressible case.

The most interesting results, however, involve $\mathcal{F}\left\{\mathrm{T} 3_{1}\right\}$ and $\mathcal{F}\{\mathrm{T} 5\}$. First consider the former. Noting that, from the definition of $r_{k}$ given before,

$$
\frac{\partial}{\partial r_{k}}=\frac{1}{2}\left[\left(\frac{\partial}{\partial x_{k}}\right)_{B}-\left(\frac{\partial}{\partial x_{k}}\right)_{A}\right]
$$

the term $\mathrm{T} 3_{1}$ from Eq. 3.12 , in the limit $\overrightarrow{\mathrm{r}}=0$, can be written as

$$
\begin{aligned}
{\left[\mathrm{T} 3_{1}\right]_{\mathrm{r}=0}=\left\{\frac { 1 } { 2 } \left[\left(\frac{\partial}{\partial x_{k}}\right)_{B}\right.\right.} & \left.-\left(\frac{\partial}{\partial x_{k}}\right)_{A}\right]\left(\overline{\left(u_{i}^{\prime \prime}\right)_{A}\left(\rho u_{j}^{\prime \prime} u_{k}^{\prime \prime}\right)_{B}}-\overline{\left(\rho u_{i}^{\prime \prime} u_{k}^{\prime \prime}\right)_{A}\left(u_{j}^{\prime \prime}\right)_{B}}\right) \\
& \left.+\left(\overline{\left(\rho u_{i}^{\prime \prime}\right)_{A}\left(u_{j}^{\prime \prime} u_{k}^{\prime \prime}\right)_{B}}-\overline{\left(u_{i}^{\prime \prime} u_{k}^{\prime \prime}\right)_{A}\left(\rho u_{j}^{\prime \prime}\right)_{B}}\right)\right\}_{A=B}=0 .
\end{aligned}
$$

Introducing the inverse Fourier transform operator

$$
\mathcal{F}^{-1}\{. .\}=\iint_{-\infty}^{\infty} \exp (i \overrightarrow{\mathrm{k}} \cdot \overrightarrow{\mathrm{r}})(. .) d \overrightarrow{\mathrm{k}}
$$

this can also be expressed as

$$
\left[\mathrm{T} 3_{1}\right]_{\overrightarrow{\mathrm{r}}=0}=\mathcal{F}^{-1}\left\{\mathcal{F}\left\{T 3_{1}\right\}\right\}_{\overrightarrow{\mathrm{r}}=0}=\iint_{-\infty}^{\infty} \int_{\infty} \mathcal{F}\left\{T 3_{1}\right\} d \overrightarrow{\mathrm{k}}=0 .
$$

This relation implies that the term $\mathcal{F}\left\{\mathrm{T} 3_{1}\right\}$ when integrated over all wavenumbers is identically zero. In other words this term is not a net source or sink in the balance of t.k.e. but merely redistributes energy across wavenumbers. This is a significant result as, in effect, it states that the density-weighted triple velocity correlations do not produce or destroy density-weighted turbulent kinetic energy, but merely transfer it between wavenumbers, a characteristic shared by the unweighted triple velocity correlations in the incompressible case (Hinze 1975).

As for the term $\mathcal{F}\{\mathrm{T} 5\}$ it can be split into two terms, one involving mean pressure and the other involving fluctuating pressure. In the absence of externally imposed pressure 
gradients, the former is expected to be negligible. The latter comprises $\mathrm{T} 55_{1}$ which is often referred to as a pressure-dilatation term in the context of single-point correlations since the contraction of indices in Eq. 3.12 makes the fluctuating part of T5

$$
\frac{\partial}{\partial r_{i}}\left[\overline{p_{A}^{\prime}\left(u_{i}^{\prime \prime}\right)_{B}}-\overline{p_{B}^{\prime}\left(u_{i}^{\prime \prime}\right)_{A}}\right]
$$

Hinze (1975) shows that in incompressible isotropic turbulence the condition $\partial u_{i}^{\prime \prime} / \partial x_{i}=0$ leads to the fluctuating pressure-velocity correlation tensor, $\overline{p_{A}^{\prime}\left(u_{i}^{\prime \prime}\right)_{B}}$, becoming zero. Hence $\mathcal{F}\{\mathrm{T} 5\}$ does not appear in the final energy balance. This is clearly not the case in compressible flows. It is well known (Zeman 1991; Sarkar et al. 1991) that in non-reacting compressible flows at moderate to high Mach numbers, pressure-dilatation has a leading order influence in the balance of $\widetilde{t . k . e}$., particularly if shocklets arise in the flow. On the other hand, in low speed reactive flows pressure-dilatation has been shown to be a major source of $\widehat{t . k . e}$. (Zhang \& Rutland 1995). Our analysis suggests that even when the energy balance is considered in wavenumber space, pressure-velocity correlations can be interpreted as a net source or sink. More significantly, dilatation fluctuations mostly occur at flame scales, typically at the high wavenumber end of the energy spectrum. Therefore, pressure-velocity correlations could be a significant source of backscatter. The analysis presented here provides a formal mathematical framework to quantify, and possibly model, the extent of backscatter using dimensional arguments.

By analogy with the energy balance, the density-weighted two-point scalar correlation balance can similarly be derived. While the primary interest is in correlations of a scalar with itself, the equation is derived for the more general case of correlations between two different scalars, $\psi$ and $\xi$. Starting from the balance equation for $\psi^{\prime \prime}$, Eq. B 1 derived in appendix B,

$$
\frac{\partial \psi^{\prime \prime}}{\partial t}+\frac{\partial \widetilde{\psi}}{\partial t}+\widetilde{U}_{k} \frac{\partial \widetilde{\psi}}{\partial x_{k}}+u_{k}^{\prime \prime} \frac{\partial \widetilde{\psi}}{\partial x_{k}}+\widetilde{U}_{k} \frac{\partial \psi^{\prime \prime}}{\partial x_{k}}+u_{k}^{\prime \prime} \frac{\partial \psi^{\prime \prime}}{\partial x_{k}}=\frac{\dot{\omega}_{\psi}}{\rho}+\frac{\mathcal{D}_{\psi}}{\rho}
$$

and that for $\rho \psi^{\prime \prime}$, Eq. B 4,

$$
\frac{\partial \rho \psi^{\prime \prime}}{\partial t}+\rho^{\prime} \frac{\partial \widetilde{\psi}}{\partial t}+\rho^{\prime} \widetilde{U}_{k} \frac{\partial \widetilde{\psi}}{\partial x_{k}}+\rho u_{k}^{\prime \prime} \frac{\partial \widetilde{\psi}}{\partial x_{k}}+\frac{\partial \rho \psi^{\prime \prime} \widetilde{U}_{k}}{\partial x_{k}}+\frac{\partial}{\partial x_{k}}\left(\rho u_{k}^{\prime \prime} \psi^{\prime \prime}-\overline{\rho u_{k}^{\prime \prime} \psi^{\prime \prime}}\right)=\dot{\omega}_{\psi}^{\prime}+\mathcal{D}_{\psi}^{\prime}
$$

We multiply the equation for $\left(\psi^{\prime \prime}\right)_{A}$ by $\left(\rho \xi^{\prime \prime}\right)_{B}$ and the equation for $\left(\xi^{\prime \prime}\right)_{B}$ by $\left(\rho \psi^{\prime \prime}\right)_{A}$. Similarly, we multiply the equations for $\left(\rho \psi^{\prime \prime}\right)_{A}$ and $\left(\rho \xi^{\prime \prime}\right)_{B}$ by $\left(\xi^{\prime \prime}\right)_{B}$ and $\left(\psi^{\prime \prime}\right)_{A}$ respec- 
tively. Adding and averaging the result yields

$$
\begin{aligned}
& \frac{2 \partial \bar{\rho} \widetilde{R}_{\psi \xi}}{\partial t}+\overline{\rho_{A}^{\prime}\left(\xi^{\prime \prime}\right)_{B}}\left(\frac{\partial \widetilde{\psi}}{\partial t}+\widetilde{U}_{k} \frac{\partial \widetilde{\psi}}{\partial x_{k}}\right)_{A}+\overline{\rho_{B}^{\prime}\left(\psi^{\prime \prime}\right)_{A}}\left(\frac{\partial \widetilde{\xi}}{\partial t}+\widetilde{U}_{k} \frac{\partial \widetilde{\xi}}{\partial x_{k}}\right)_{B} \\
& +\overline{\left(\xi^{\prime \prime}\right)_{B}\left(\rho u_{k}^{\prime \prime}\right)_{A}}\left(\frac{\partial \widetilde{\psi}}{\partial x_{k}}\right)_{A}+\overline{\left(\rho \xi^{\prime \prime}\right)_{B}\left(u_{k}^{\prime \prime}\right)_{A}}\left(\frac{\partial \widetilde{\psi}}{\partial x_{k}}\right)_{A}+\overline{\left(\psi^{\prime \prime}\right)_{A}\left(\rho u_{k}^{\prime \prime}\right)_{B}}\left(\frac{\partial \widetilde{\xi}}{\partial x_{k}}\right)_{B}+\overline{\left(\rho \psi^{\prime \prime}\right)_{A}\left(u_{k}^{\prime \prime}\right)_{B}}\left(\frac{\partial \widetilde{\xi}}{\partial x_{k}}\right)_{B} \\
& +\left(\frac{\partial}{\partial x_{k}}\right)_{A}\left(\widetilde{U}_{k}\right)_{A} \overline{\left(\rho \psi^{\prime \prime}\right)_{A}\left(\xi^{\prime \prime}\right)_{B}}+\left(\widetilde{U}_{k} \frac{\partial}{\partial x_{k}}\right)_{A} \overline{\left(\psi^{\prime \prime}\right)_{A}\left(\rho \xi^{\prime \prime}\right)_{B}}+\left(\frac{\partial}{\partial x_{k}}\right)_{B}\left(\widetilde{U}_{k}\right)_{B} \overline{\left(\rho \xi^{\prime \prime}\right)_{B}\left(\psi^{\prime \prime}\right)_{A}}+\left(\widetilde{U}_{k} \frac{\partial}{\partial x_{k}}\right)_{B} \overline{\left(\xi^{\prime \prime}\right)_{B}\left(\rho \psi^{\prime \prime}\right)_{A}} \\
& +\left(\frac{\partial}{\partial x_{k}}\right)_{A} \overline{\left(\rho u_{k}^{\prime \prime} \psi^{\prime \prime}\right)_{A}\left(\xi^{\prime \prime}\right)_{B}}+\overline{\left(\rho \xi^{\prime \prime}\right)_{B}\left(u_{k}^{\prime \prime} \frac{\partial \psi^{\prime \prime}}{\partial x_{k}}\right)_{A}}+\left(\frac{\partial}{\partial x_{k}}\right)_{B} \overline{\left(\rho u_{k}^{\prime \prime} \xi^{\prime \prime}\right)_{B}\left(\psi^{\prime \prime}\right)_{A}}+\overline{\left(\rho \psi^{\prime \prime}\right)_{A}\left(u_{k}^{\prime \prime} \frac{\partial \xi^{\prime \prime}}{\partial x_{k}}\right)_{B}} \\
& -\overline{\left(\psi^{\prime \prime}\right)_{A}}\left(\frac{\partial \overline{\rho u_{k}^{\prime \prime} \xi^{\prime \prime}}}{\partial x_{k}}\right)_{B}-\overline{\left(\xi^{\prime \prime}\right)_{B}}\left(\frac{\partial \overline{\rho u_{k}^{\prime \prime} \psi^{\prime \prime}}}{\partial x_{k}}\right)_{A}=\overline{\left(\dot{\omega}_{\psi}^{\prime}\right)_{A}\left(\xi^{\prime \prime}\right)_{B}}+\overline{\left(\dot{\omega}_{\xi}^{\prime}\right)_{B}\left(\psi^{\prime \prime}\right)_{A}} \\
& +\overline{\left(\frac{\dot{\omega}_{\psi}+\mathcal{D}_{\psi}}{\rho}\right)_{A}\left(\rho \xi^{\prime \prime}\right)_{B}}+\overline{\left(\frac{\dot{\omega}_{\xi}+\mathcal{D}_{\xi}}{\rho}\right)_{B}\left(\rho \psi^{\prime \prime}\right)_{A}}+\overline{\left(\mathcal{D}_{\psi}^{\prime}\right)_{A}\left(\xi^{\prime \prime}\right)_{B}}+\overline{\left(\mathcal{D}_{\xi}^{\prime}\right)_{B}\left(\psi^{\prime \prime}\right)_{A}} .
\end{aligned}
$$

Analogous to Eq. 3.11, the second and sixteenth terms can be simplified using Eq. B 3 as

$$
\overline{\left(\rho_{A}^{\prime}-\rho_{B}^{\prime}\right)\left(\xi^{\prime \prime}\right)_{B}}\left(\frac{\partial \widetilde{\psi}}{\partial t}+\widetilde{U}_{k} \frac{\partial \widetilde{\psi}}{\partial x_{k}}\right)_{A}-\overline{\left(\xi^{\prime \prime}\right)_{B}}\left(\overline{\left(\dot{\omega}_{\psi}\right)_{A}}+\overline{\left(\mathcal{D}_{\psi}\right)_{A}}\right)
$$

and the third and seventeenth terms as

$$
\overline{\left(\rho_{B}^{\prime}-\rho_{A}^{\prime}\right)\left(\psi^{\prime \prime}\right)_{A}}\left(\frac{\partial \widetilde{\xi}}{\partial t}+\widetilde{U}_{k} \frac{\partial \widetilde{\xi}}{\partial x_{k}}\right)_{B}-\overline{\left(\psi^{\prime \prime}\right)_{A}}\left(\overline{\left(\dot{\omega}_{\xi}\right)_{B}}+\overline{\left(\mathcal{D}_{\xi}\right)_{B}}\right) .
$$

Similarly, terms eight through eleven simplify to

$$
\left[\overline{\left(\psi^{\prime \prime}\right)_{A}\left(\rho \xi^{\prime \prime}\right)_{B}}-\overline{\left(\rho \psi^{\prime \prime}\right)_{A}\left(\xi^{\prime \prime}\right)_{B}}\right] \frac{\partial \widetilde{U}_{k}}{\partial r_{k}}
$$

while terms twelve through fifteen to

$$
\begin{aligned}
-\frac{\partial}{\partial r_{k}}\left[\overline{\left(\rho \psi^{\prime \prime} u_{k}^{\prime \prime}\right)_{A}\left(\xi^{\prime \prime}\right)_{B}}+\overline{\left(\psi^{\prime \prime} u_{K}^{\prime \prime}\right)_{A}\left(\rho \xi^{\prime \prime}\right)_{B}}\right]+\frac{\partial}{\partial r_{k}}\left[\overline{\left(\rho \psi^{\prime \prime}\right)_{A}\left(u_{k}^{\prime \prime} \xi^{\prime \prime}\right)_{B}}+\overline{\left(\psi^{\prime \prime}\right)_{A}\left(\rho \xi^{\prime \prime} u_{k}^{\prime \prime}\right)_{B}}\right] & \overline{\left(\rho \xi^{\prime \prime}\right)_{B}\left(\psi^{\prime \prime} \frac{\partial u_{k}^{\prime \prime}}{\partial x_{k}}\right)_{A}} \overline{-\left(\rho \psi^{\prime \prime}\right)_{A}\left(\xi^{\prime \prime} \frac{\partial u_{k}^{\prime \prime}}{\partial x_{k}}\right)_{B}} .
\end{aligned}
$$


Finally, Eq. 3.15 simplifies to

$$
\begin{aligned}
& \frac{2 \partial \bar{\rho} \widetilde{R}_{\psi \xi}}{\partial t}-\underbrace{\left[\overline{\left(\rho u_{k}^{\prime \prime}\right)_{A}\left(\xi^{\prime \prime}\right)_{B}}+\overline{\left(u_{k}^{\prime \prime}\right)_{A}\left(\rho \xi^{\prime \prime}\right)_{B}}\right]\left(\frac{\partial \widetilde{\psi}}{\partial r_{k}}\right)+\left[\overline{\left(\rho u_{k}^{\prime \prime}\right)_{B}\left(\psi^{\prime \prime}\right)_{A}}+\overline{\left(u_{k}^{\prime \prime}\right)_{B}\left(\rho \psi^{\prime \prime}\right)_{A}}\right]\left(\frac{\partial \widetilde{\xi}}{\partial r_{k}}\right)}_{\mathrm{T} 1} \\
& -\underbrace{\left[\overline{\left(\psi^{\prime \prime}\right)_{A}\left(\rho \xi^{\prime \prime}\right)_{B}}-\overline{\left(\rho \psi^{\prime \prime}\right)_{A}\left(\xi^{\prime \prime}\right)_{B}}\right]\left(\frac{\partial \widetilde{U}_{k}}{\partial r_{k}}\right)}_{\mathrm{T} 2} \\
& -\underbrace{\frac{\partial}{\partial r_{k}}\left[\overline{\left(\rho \psi^{\prime \prime} u_{k}^{\prime \prime}\right)_{A}\left(\xi^{\prime \prime}\right)_{B}}+\overline{\left(\psi^{\prime \prime} u_{K}^{\prime \prime}\right)_{A}\left(\rho \xi^{\prime \prime}\right)_{B}}\right]+\frac{\partial}{\partial r_{k}}\left[\overline{\left(\rho \psi^{\prime \prime}\right)_{A}\left(u_{k}^{\prime \prime} \xi^{\prime \prime}\right)_{B}}+\overline{\left(\psi^{\prime \prime}\right)_{A}\left(\rho \xi^{\prime \prime} u_{k}^{\prime \prime}\right)_{B}}\right]}_{\text {T3 } 3_{1}} \\
& \underbrace{\overline{-\left(\rho \xi^{\prime \prime}\right)_{B}\left(\psi^{\prime \prime} \frac{\partial u_{k}^{\prime \prime}}{\partial x_{k}}\right)_{A}}-\overline{\left(\rho \psi^{\prime \prime}\right)_{A}\left(\xi^{\prime \prime} \frac{\partial u_{k}^{\prime \prime}}{\partial x_{k}}\right)_{B}}}_{\text {T3 } 2}+\underbrace{\overline{\left(\rho_{A}^{\prime}-\rho_{B}^{\prime}\right)\left(\xi^{\prime \prime}\right)_{B}}\left(\frac{\partial \widetilde{\psi}}{\partial t}+\widetilde{U}_{k} \frac{\partial \widetilde{\psi}}{\partial x_{k}}\right)_{A}+\overline{\left(\rho_{B}^{\prime}-\rho_{A}^{\prime}\right)\left(\psi^{\prime \prime}\right)_{A}}\left(\frac{\partial \widetilde{\xi}}{\partial t}+\widetilde{U}_{k} \frac{\partial \widetilde{\xi}}{\partial x_{k}}\right)_{B}}_{\text {T4 }} \\
& =\underbrace{\overline{\left(\dot{\omega}_{\psi}\right)_{A}\left(\xi^{\prime \prime}\right)_{B}}+\overline{\left(\dot{\omega}_{\xi}\right)_{B}\left(\psi^{\prime \prime}\right)_{A}}}_{\mathrm{T} 5_{1}} \underbrace{+\overline{\left(\dot{\omega}_{\psi} / \rho\right)_{A}\left(\rho \xi^{\prime \prime}\right)_{B}}+\overline{\left(\dot{\omega}_{\xi} / \rho\right)_{B}\left(\rho \psi^{\prime \prime}\right)_{A}}}_{\mathrm{T} 5_{2}} \\
& +\underbrace{\overline{\left(\mathcal{D}_{\psi}\right)_{A}\left(\xi^{\prime \prime}\right)_{B}}+\overline{\left(\mathcal{D}_{\xi}\right)_{B}\left(\psi^{\prime \prime}\right)_{A}}}_{\mathrm{T} 6_{1}} \underbrace{+\overline{\left(\mathcal{D}_{\psi} / \rho\right)_{A}\left(\rho \xi^{\prime \prime}\right)_{B}}+\overline{\left(\mathcal{D}_{\xi} / \rho\right)_{B}\left(\rho \psi^{\prime \prime}\right)_{A}}}_{\mathrm{T} 6_{2}} .
\end{aligned}
$$

The similarities between Eqs. 3.16 and 3.12 are obvious. Likewise, the physical interpretation of various terms in 3.16 is also similar to those in Eq. 3.12, with only subtle differences: T1 represents the contribution of mean scalar gradients; T2 represents the interaction between mean flow and scalar-scalar correlations; T3 represents the contribution of scalar-scalar-velocity triple correlations; T4 represents the influence of fluctuating density coupled with mean velocity and mean scalar gradients; T5 represents the contributions of chemical reactions and T6 represents the dissipative terms due to molecular diffusion. Also, it can be readily shown that, upon applying the Fourier transform operation, the term $\mathrm{T} 3_{1}$ will be identically zero when integrated over all wavenumbers, and hence, it has the same role as T3 ${ }_{1}$ in Eq. 3.12 i.e. transferring scalar fluctuations between wavenumbers. Also, the role of chemical reactions is now simply encapsulated in term T5. In some respects it can be considered analogous to the pressure-velocity correlation term in Eq. 3.12, since both terms are expected to be most significant at high wavenumbers corresponding to chemical scales.

\section{Results}

As evident from the description of the DNS database, the simulations correspond to a statistically one-dimensional case with moments varying only in the transverse direction and in time. Accordingly, spectra are constructed from a fixed temporal snapshot and at fixed transverse distance relative to the mid-plane. Furthermore statistical symmetry about the mid-plane is exploited and samples from equi-distant positive and negative transverse planes are considered as statistically equivalent. It is convenient to map the transverse distance to a mean progress variable since the latter readily refers to a location in the flame brush. The progress variable, $c$, is defined based on normalised oxygen mass 

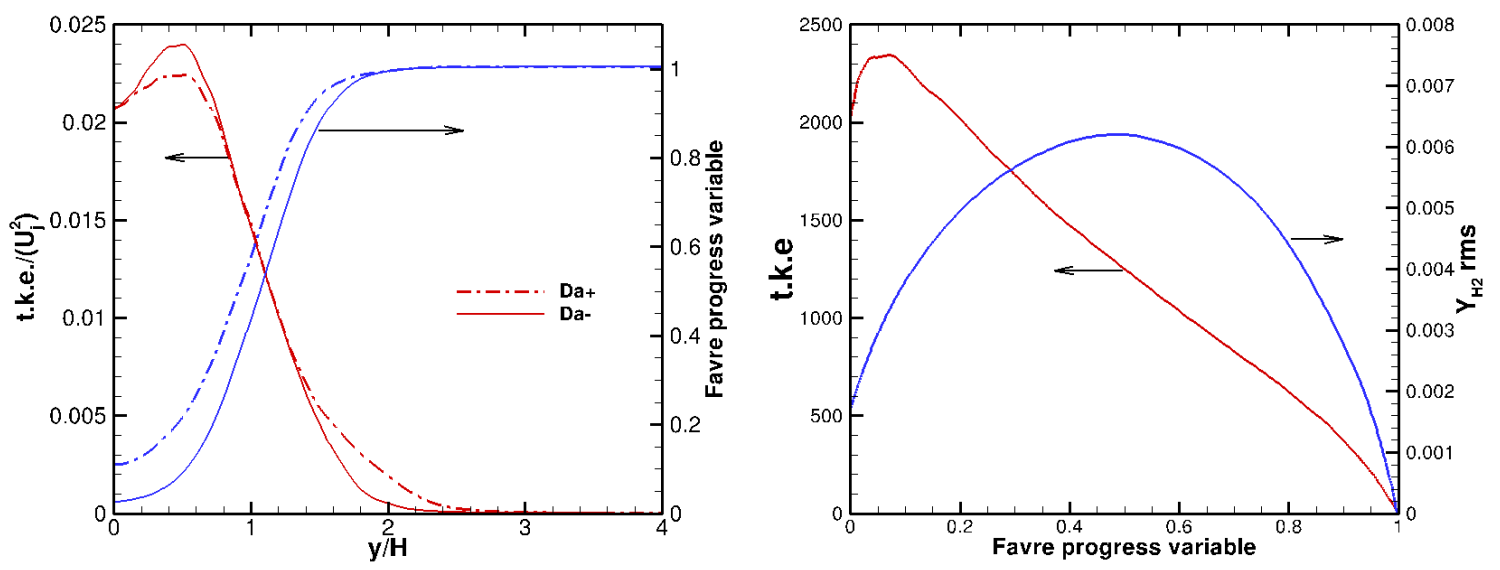

Figure 3. The typical one-dimensional flame brush from the DNS. The plot on left shows Favre turbulent kinetic energy, $\widetilde{t . k . e}$., normalised by $U_{j}^{2}$ and Favre progress variable, $\widetilde{c}$, as a function of normalised transverse coordinate, $y / H$, for the $\mathrm{Da}$ - (solid) and $\mathrm{Da}+$ (dashed) cases at $t / t_{j}=15$. The plot on right shows $\widetilde{t . k . e}$. (red) and Favre fluctuations of hydrogen mass fraction (blue) plotted against $\tilde{c}$ for the Da- case at $t / t_{j}=15$.

fraction as

$$
c \equiv \frac{Y_{O_{2}, u}-Y_{O_{2}}}{Y_{O_{2}, u}-Y_{O_{2}, b}},
$$

such that it assumes the value zero in the unburnt mixture, denoted by subscript $u$, and unity in the fully burnt mixture, denoted by subscript $b$. The progress variable could alternatively be defined using mass fractions of hydrogen or water. Oxygen was chosen here because it is the excess reactant and has non-zero values both on the unburnt and fully burnt regions and hence the definition is numerically cleaner. Figure 3 shows the typical flame brush, in physical as well as progress variable space, from the Da- and Da+ cases for the temporal snapshot corresponding to $t / t_{j}=15$. Since the streamwise and spanwise homogeneous directions exist the spectra could be constructed as a function of wavenumber magnitude, $k$, either by performing two-dimensional Fourier transforms and averaging all ensembles of equal $k$, or by performing one-dimensional Fourier transforms along one direction and averaging along the other direction. Here the latter is adopted for two reasons. The numerical convergence of the one-dimensional transforms is better since there are a sufficient number of samples for a given value of $k$ to perform the ensemble averaging. For two-dimensional transforms, since the grid is rectangular Cartesian, the number of samples becomes progressively smaller with decreasing $k$. The second reason is that the domain size, and hence the length scale of periodicity, is different in the two directions and therefore the two-dimensional transforms are slightly ambiguous. As a consequence of the density weighting in the definitions of correlation tensors and spectrum functions, the one-dimensional spectrum, of say velocity component $u_{1}^{\prime \prime}$, is computed as

$$
\begin{aligned}
E_{u_{1}} & =\frac{1}{2 \bar{\rho}}\left(\overline{\mathcal{F}\left\{\rho u_{1}^{\prime \prime}\right\} \mathcal{F}^{*}\left\{u_{1}^{\prime \prime}\right\}}+\overline{\mathcal{F}\left\{u_{1}^{\prime \prime}\right\} \mathcal{F}^{*}\left\{\rho u_{1}^{\prime \prime}\right\}}\right) \\
& =\frac{1}{2 \bar{\rho}}\left[\frac{1}{2 N_{z}} \sum_{k=1}^{2 N_{z}}\left(\mathcal{F}_{k}\left\{\rho u_{1}^{\prime \prime}\right\} \mathcal{F}_{k}^{*}\left\{u_{1}^{\prime \prime}\right\}+\mathcal{F}_{k}\left\{u_{1}^{\prime \prime}\right\} \mathcal{F}_{k}^{*}\left\{\rho u_{1}^{\prime \prime}\right\}\right)\right],
\end{aligned}
$$




\begin{tabular}{l|ccc|ccc}
\hline & \multicolumn{3}{|c}{ Da- } & \multicolumn{3}{c}{ Da+ } \\
\hline$\tilde{c}$ & 0.1 & 0.5 & 0.9 & 0.1 & 0.5 & 0.9 \\
\hline $\mathrm{Re}_{\mathrm{t}}$ & 428.4 & 90.8 & 15.7 & 354.2 & 103.9 & 19.4 \\
$\mathrm{Re}_{\lambda}$ & 80.2 & 36.9 & 15.3 & 72.9 & 39.5 & 17.1 \\
$\mathrm{Ka}$ & 0.71 & 0.2 & 0.05 & 0.16 & 0.05 & 0.02 \\
$\tilde{\eta} * k_{\max }$ & 2.2 & 4.15 & 8.44 & 2.33 & 4.0 & 6.9
\end{tabular}

TABLE 2. Relevant DNS parameters of the cases for which spectra are studied.

where $\mathcal{F}_{k}$ and $\mathcal{F}_{k}^{*}$ denote the Fourier transform and its complex conjugate, respectively, of each streamwise line signal from the two equi-distant planes, and $2 N_{z}$ is the total number of line samples. The mean density, $\bar{\rho}$, is computed from an average over both planes.

\subsection{Turbulence kinetic energy spectra}

Figure 4 shows a sample one-dimensional kinetic energy spectrum, $E^{1 D} \equiv 0.5\left(E_{u_{1}}+\right.$ $\left.E_{u_{2}}+E_{u_{3}}\right)$, from the Da+ case corresponding to transverse planes of $\widetilde{c}=0.5$ at time $t=15 t_{j}$. It is evident that there is a large range of dynamic scales, with a clear inertial range, attesting to the high quality of the DNS dataset. The statistical convergence is assessed on the plot on the left by comparing the spectrum from the full set of samples with that constructed from samples only from the bottom plane, $y=-4.9 \mathrm{~mm}$, and that constructed from samples only from the top plane, $y=4.9 \mathrm{~mm}$, (see Fig. 1). The two planes are separated by a distance of $9.8 \mathrm{~mm}$ which is over six times greater than the integral scale, $\Lambda$, which is $1.49 \mathrm{~mm}$ for this case. Hence the samples from the bottom plane can be deemed statistically independent from those on the top plane. As is evident the three spectra are barely distinguishable indicating statistical convergence. Also shown is the comparison between the density-weighted and the conventional unweighted spectra. The latter is constructed as described in Eq. 4.2, but without the density terms. It is interesting to note that including the density weighting does not seem to influence the spectrum in the low wavenumber (energy-containing and inertial) range, but a difference is apparent in the high wavenumber range. The latter is traditionally referred to as the dissipative range, but as the ensuing discussion will show shortly, it might more appropriately be viewed instead as a "reactive-dissipative" range in the current case. This comparison should not be viewed as the efficacy, or lack thereof, of the density weighting in the spectra definitions. It is likely that factors such as the density jump across the premixed flame front (which is relatively low for the present preheated lean hydrogen cases) and the wavenumber characteristic of density fluctuations will determine the extent of the differences that arise due to the density-weighted definition. Furthermore, as we showed in section 3 , the density weighting makes the interpretation of the spectrum balance equations much simpler and more meaningful.

It is worth commenting on some numerical aspects that can influence computation of the spectra. As mentioned earlier the DNS employs a tenth-order filter to improve stability. The filter, described in detail by Kennedy \& Carpenter (1994), is an explicit central-difference filter and is purely dissipative. The spectral transfer function of the filter, shown as a dashed line in Fig. 4, indicates that it is monotonic and confirms its dissipative nature at high wavenumbers. Consequently the filter will only remove energy at these high wavenumbers and will not add energy spuriously. Another potential numerical artifact concerns aliasing. It was shown in Hawkes et al. (2012) that for the temporal snapshots being considered $\left(t / t_{j}=15\right)$ the mean Kolmogorov length scale is 
greater than at least half the grid size, $\widetilde{\eta}>0.5 \Delta x$, which is considered sufficient resolution for turbulent flow DNS (Yeung \& Pope 1989). Hence we believe the contribution from aliasing errors is negligible in the current results. And finally, it is a conventional practice to pre-multiply signals with windowing functions before performing a discrete Fourier transform to avoid spectral leakages. In the present study however, no windowing functions were employed since the 1D line signals on which the Fourier transform was performed were guaranteed to be periodic owing to the DNS boundary conditions.

The one-dimensional spectra from the Da- and Da+ cases at three locations spanning the flame brush $-\tilde{c}=0.1,0.5$ and 0.9 - are shown in Fig. 5. The relevant non-dimensional DNS parameters for these locations are listed in Table 2 . These parameters are calculated using the following expressions

$$
\begin{array}{r}
\text { turbulent Reynolds number, } \operatorname{Re}_{\mathrm{t}}=\frac{u^{\prime \prime} \Lambda}{\widetilde{\nu}}, \\
\text { Taylor scale Reynolds number, } \operatorname{Re}_{\lambda_{\mathrm{g}}}=\frac{u^{\prime \prime} \lambda_{g}}{\widetilde{\nu}}, \\
\text { Karlovitz number, } \mathrm{Ka}=\left(\frac{\nu_{u} / s_{L}}{\widetilde{\eta}}\right)^{2},
\end{array}
$$

where the integral length and velocity scales, $\Lambda$ and $u^{\prime \prime}$ respectively, the Taylor microscale, $\lambda_{g}$, and the Kolmogorov scale, $\widetilde{\eta}$, are evaluated using

$$
\begin{array}{r}
u^{\prime \prime}=(2 \widetilde{t . k . e} / 3)^{1 / 2}, \Lambda=u^{\prime \prime 3} / \widetilde{\epsilon} \\
\lambda_{g}=\left(15 \widetilde{\nu} u^{\prime \prime 2} / \widetilde{\epsilon}\right)^{1 / 2} \text { and } \widetilde{\eta}=\left(\widetilde{\nu}^{3} / \widetilde{\epsilon}\right)^{1 / 4} .
\end{array}
$$

Note that Favre averaged kinematic viscosity is $\widetilde{\nu}$ and Favre averaged turbulent kinetic energy dissipation rate is $\widetilde{\epsilon}$. The parameter $\widetilde{\eta} * k_{\max }$ in Table 2 , where $k_{\max }$ is the Nyquist wavenumber, denotes the level of resolution of the smallest flow scales. Figure 5 reveals the classical spectrum shape with distinct energy containing, inertial and dissipative ranges is evident for all the cases. It is interesting to note the high wavenumber shape of the spectra. While the shape at high $k$ for most part resembles the exponential drop-off characteristic of a typical dissipation range, an unmistakeable inflection occurring in the vicinity of $k \approx 10^{5}$ in the Da- and $k \approx 5 \times 10^{4}$ in the Da+ cases is present. The temporal evolution of the shear layer and the flame brush are such that in both cases the peak $\widetilde{\text { t.k.e. }}$ and turbulent Reynolds numbers, $\mathrm{Re}_{\mathrm{t}}$, occurred at locations where $\tilde{c}$ is just less than 0.1 (see Fig. 3). Hence the values of $\widetilde{t . k . e}$. and $\mathrm{Re}_{\mathrm{t}}$ progressively decrease from $\widetilde{c}$ of 0.1 to 0.9 , a trend clearly evident in the spectra. This trend differs from that of Furukawa et al. (2002) who construct kinetic energy spectra conditioned on fresh versus burnt gas fluid and report that the turbulent kinetic energy conditioned on the burnt gas is higher than that of the fresh gas indicating flame generated turbulence. This difference is likely due to two reasons. First, the flames investigated by Furukawa et al. (2002) have much higher Damköhler numbers $(\mathcal{O}(100))$ compared to the present flames $(\mathcal{O}(0.1))$ and hence they could be in different vastly different regimes. Second, the conditional sampling used to construct the spectra by Furukawa et al. (2002) ensures that the velocity fluctuations induced by large scale flame flapping are discounted. The unconditional spectra presented here include this effect.

Knaus \& Pantano (2009) report kinetic energy spectra from DNS of a non-premixed flame in a temporally evolving shear layer, a configuration nearly identical to the present DNS. They find a surprisingly good scaling of the spectra when normalised using Favre averaged quantities. They examined the scaling of the normalised one-dimensional energy 

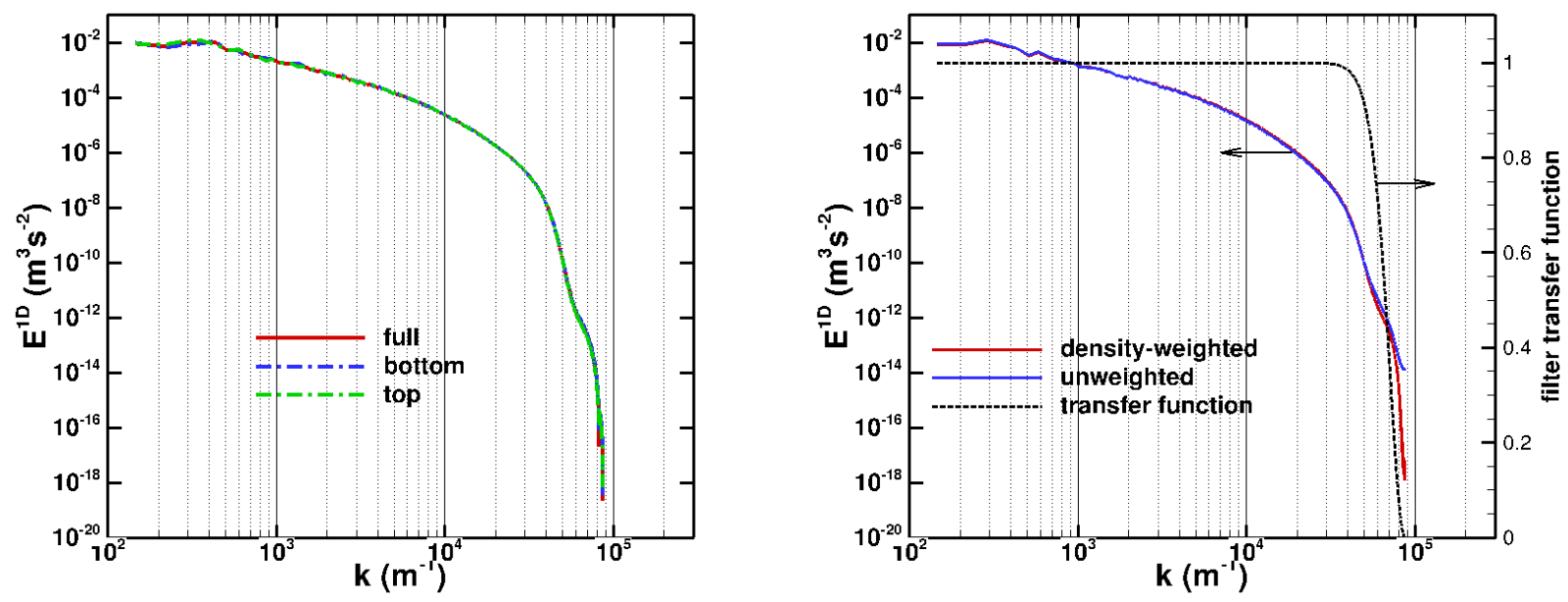

FiguRE 4. A sample one-dimensional turbulent kinetic energy spectrum for the Da+ case corresponding to $\widetilde{c}=0.5$ at time $t=15 t_{j}$. The plot on the left compares the spectra constructed with the full set of samples (red) with that constructed using only samples from the bottom plane (blue) and samples only from the top plane (green). Since the curves coincide the former is plotted as a solid line and the latter using dashed lines clarity. The plot on the right compares the spectra constructed with (red) and without (blue) the density-weighted definitions, along with the transfer function (dashed) for the tenth-order filter used in the DNS.
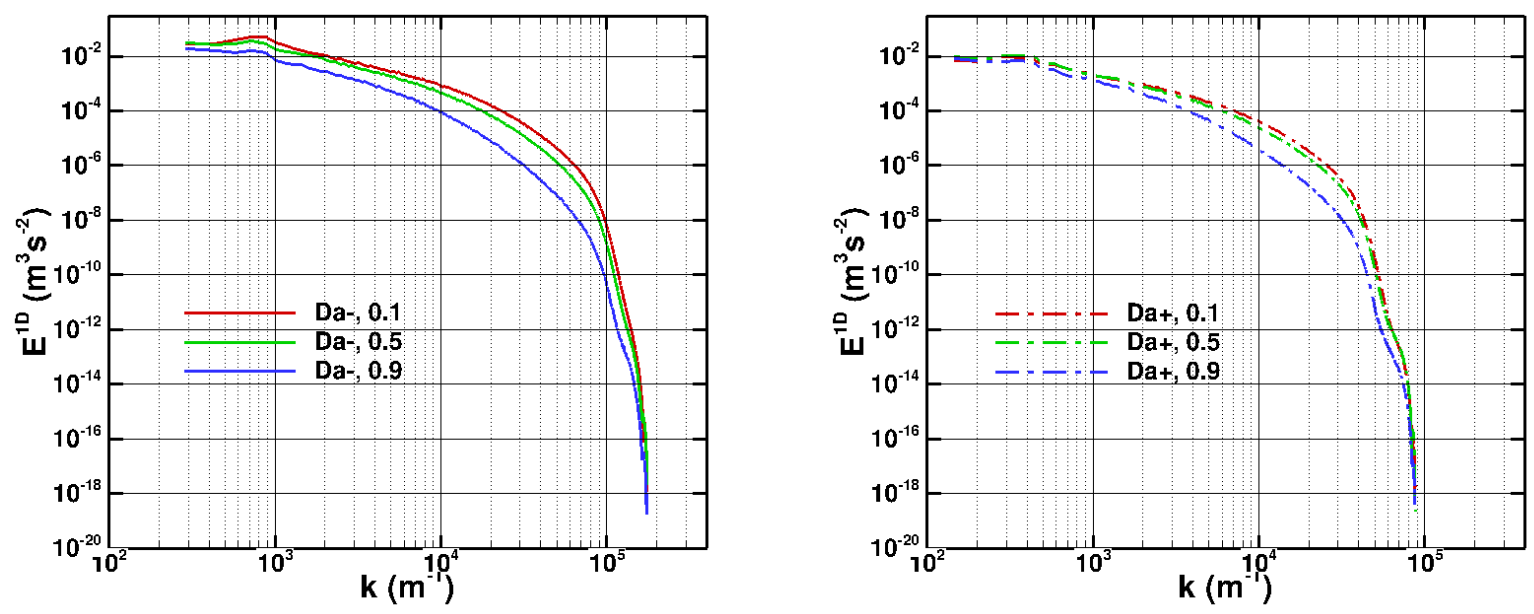

FigurE 5. The one-dimensional turbulent kinetic energy spectra for the Da- (left) and Da+ (right) cases corresponding to three flame brush locations, $\widetilde{c}=0.1$ (red), 0.5 (green), 0.9 (blue).

spectra,

$$
E_{\text {norm }}^{1 D} \equiv \widetilde{\epsilon}^{-2 / 3} \widetilde{\eta}^{-5 / 3} E(k),
$$

as a function of the normalised wavenumber, $k \widetilde{\eta}$. The rationale behind expecting such a scaling is that the Kolmogorov hypotheses yields for energy spectra a function of the 

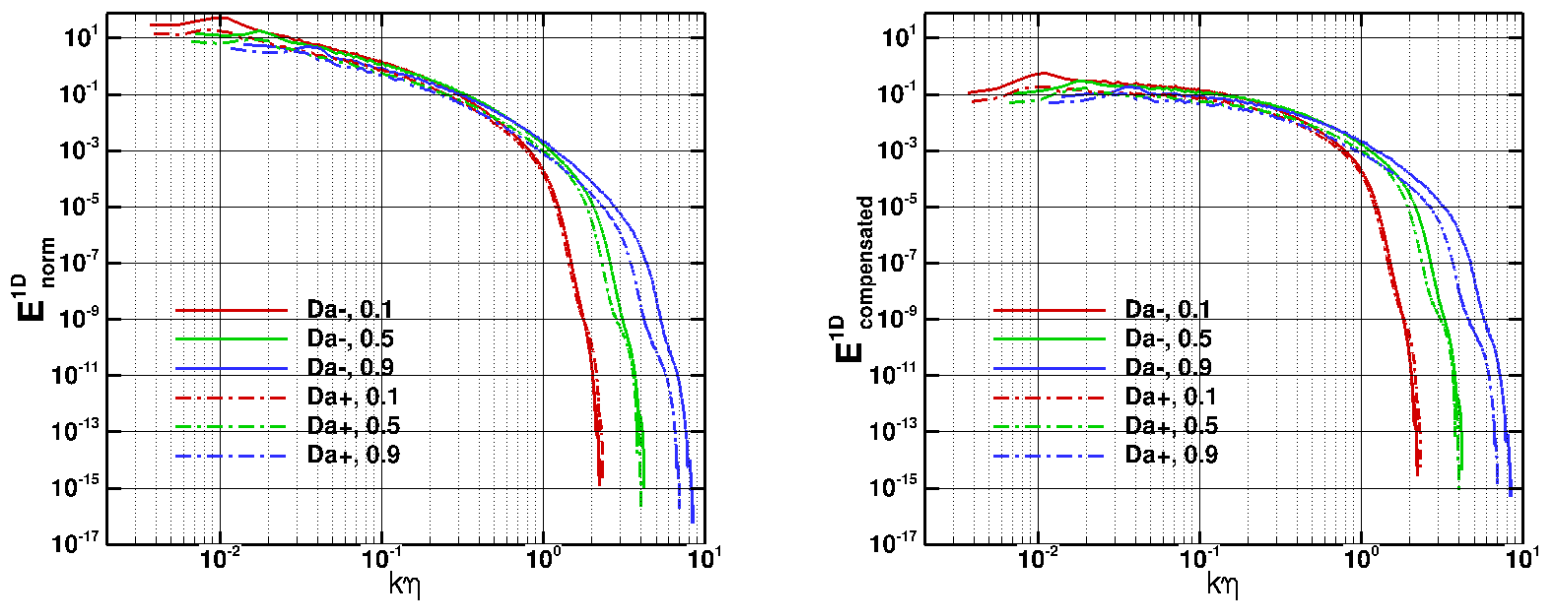

Figure 6. Plot on the left shows normalised one-dimensional turbulent kinetic energy spectra plotted against the normalised wavenumber for all locations in the Da- and Da+ cases. The plot on the right shows the compensated kinetic energy spectra.

form

$$
E(k)=C \epsilon^{2 / 3} k^{-5 / 3} f(k \eta),
$$

with the function $f(k \eta)$ capturing the exponential, presumably universal, high wavenumber drop-off. A good collapse based on Favre averaged quantities led Knaus \& Pantano (2009) to suggest that spectra in reacting flows follow the conventional Kolmogorov scaling in terms of Favre averaged quantities, an encouraging prospect. We examine this scaling by plotting the normalised energy spectra against the normalised wavenumber, for all cases in Fig. 6 . The compensated spectra for the corresponding cases, $(k \widetilde{\eta})^{5 / 3} \times E_{\text {norm }}^{1 D}$, are shown in the same figure on the right. At the outset, the compensated spectra show the presence of nearly one decade of an inertial range with a $-5 / 3$ scaling in almost all the cases, except for $\mathrm{Da}+\widetilde{c}=0.9$, which has the lowest $\mathrm{Re}_{\mathrm{t}}$ of all. More interestingly, the normalised spectra show a very good collapse in the inertial range, but deviate noticeably in the dissipative range. Evidently, a Kolmogorov type scaling involving Favre averaged quantities is not applicable over the entire wavenumber range for the current cases. Also, the value of the Kolmogorov scaling constant ( $C$ in Eq. 4.7), which can be inferred from the inertial range plateau in the compensated spectra, is around 0.2 for the current cases, which is somewhat lower than that reported by Knaus \& Pantano (2009) for non-premixed flames. However, there is possibly some uncertainty in assuming that Favre averaged quantities, $\widetilde{\epsilon}$ and $\widetilde{\eta}$, will yield a perfect Kolmogorov scaling. While the turbulent quantities, and spectra, vary significantly in space, the temporal evolution is considerably slower. Figure 7 shows the raw and normalised kinetic energy spectra from three temporal snapshots: $t / t_{j}=12,15$ and 18, for the Da- case at the $\widetilde{c}=0.5$ location.

The discussion in section 3 on the energy balance in wavenumber space provides some intuition here. As discussed before, a major difference between the incompressible and variable density reactive flows involves the pressure-velocity correlations, term $\mathrm{T} 5$ in Eq. 3.12. We anticipate that this term could be a significant source for $\widetilde{t . k . e}$., mainly through the pressure-dilatation coupling. It is well known that premixed flame fronts are accompanied by a much stronger local dilatation due to the density jump, as compared to 

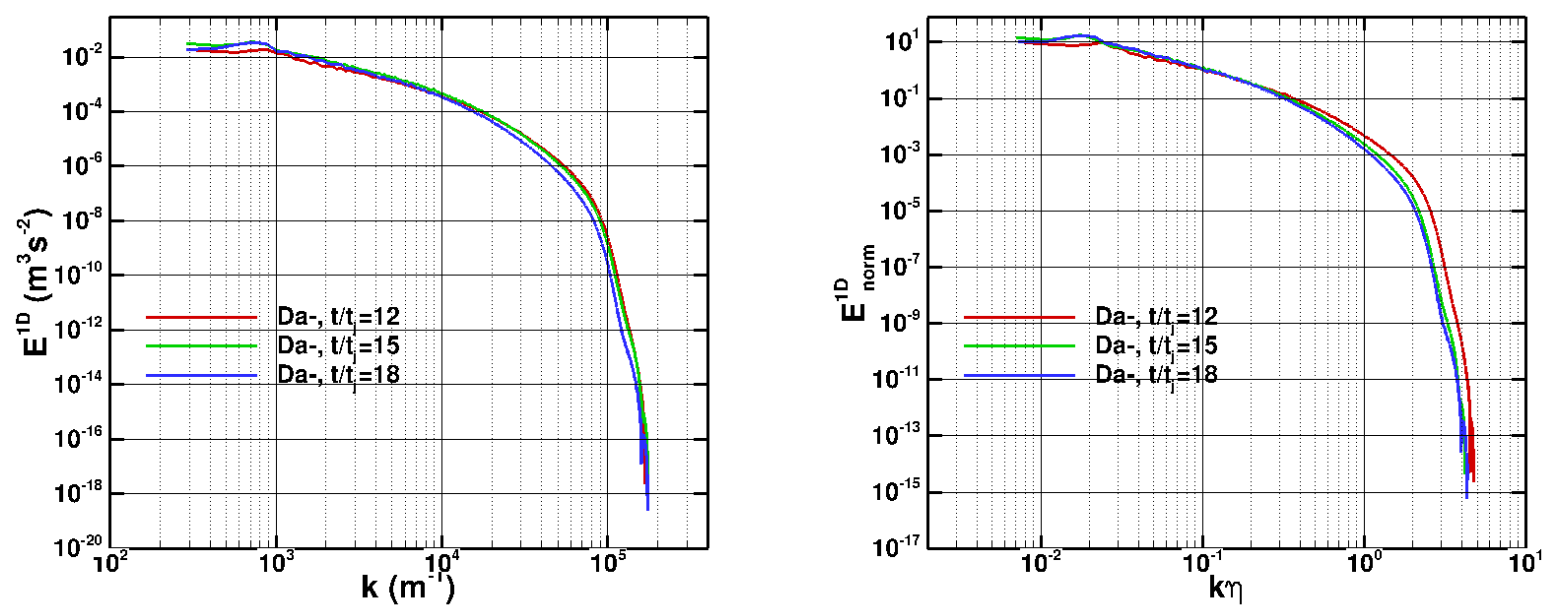

Figure 7 . Temporal evolution of one-dimensional turbulent kinetic energy spectra for the Dacase at $\widetilde{c}=0.5$ location. Plot on the left shows raw spectra from temporal snapshots corresponding to $t / t_{j}=12,15$ and 18 , and plot on the right shows corresponding normalised spectra.

non-premixed flames (Bilger 2004). The contribution of the two pressure-velocity terms, $\mathrm{T} 5_{1}$ and $\mathrm{T} 5_{2}$, are shown in Fig. 8 for the case of $\mathrm{Da}+, \widetilde{c}=0.1$. Shown in the same figure is the spectral coherence between fluctuating pressure, $p^{\prime}$, and one component of fluctuating velocity, $u_{1}^{\prime \prime}$. This figure presents strong evidence of the role of the pressurevelocity terms. Both terms are of identical magnitude at all wavenumbers and a strong 'spike' in these terms at high wavenumbers coincides nearly perfectly with the inflection in the kinetic energy spectrum. Furthermore, the fluctuating pressure and velocities seem to be significantly coherent at the same wavenumber, indicating a strong signature from the pressure-velocity correlations.

Premixed flame fronts introduce strong dilatational fluctuations at scales comparable to laminar flame thickness, and we speculate that the pressure-velocity term introduces energy into the flow at the same scales. Depending on the relative strength of the other terms in the energy balance, this energy might be transferred both forward and backward in wavenumber space. In the current simulations the unstrained laminar flame thickness, defined based on the maximum temperature gradient as

$$
\delta_{L}=\frac{T_{b}-T_{u}}{(\nabla T)_{\max }}
$$

is $5.025 \times 10^{-4} \mathrm{~m}$, while the Komogorov scale, $\widetilde{\eta}$, for all the cases lies in the range of $1.2 \times 10^{-5}$ to $8 \times 10^{-5} \mathrm{~m}$, which suggests a strong overlap of the dissipative range with the chemical scales. Accordingly, the high wavenumber range is no longer characterised only by viscous dissipation, but rather, also by the influence of chemical reactions, which in the case of kinetic energy, manifests itself through the pressure-velocity term. At the very least, the high wavenumber exponential drop-off needs to be revised to include the flame thickness $\delta_{L}$. Hence, we examine a revised normalisation of the energy spectra based on $\delta_{L}$ instead of $\widetilde{\eta}$ as

$$
E_{\text {norm }} \equiv \widetilde{\epsilon}^{-2 / 3} \delta_{L}^{-5 / 3} E(k),
$$

and plot it against a normalised wavenmber $k \delta_{L}$, as shown in Fig. 9. When thus nor- 

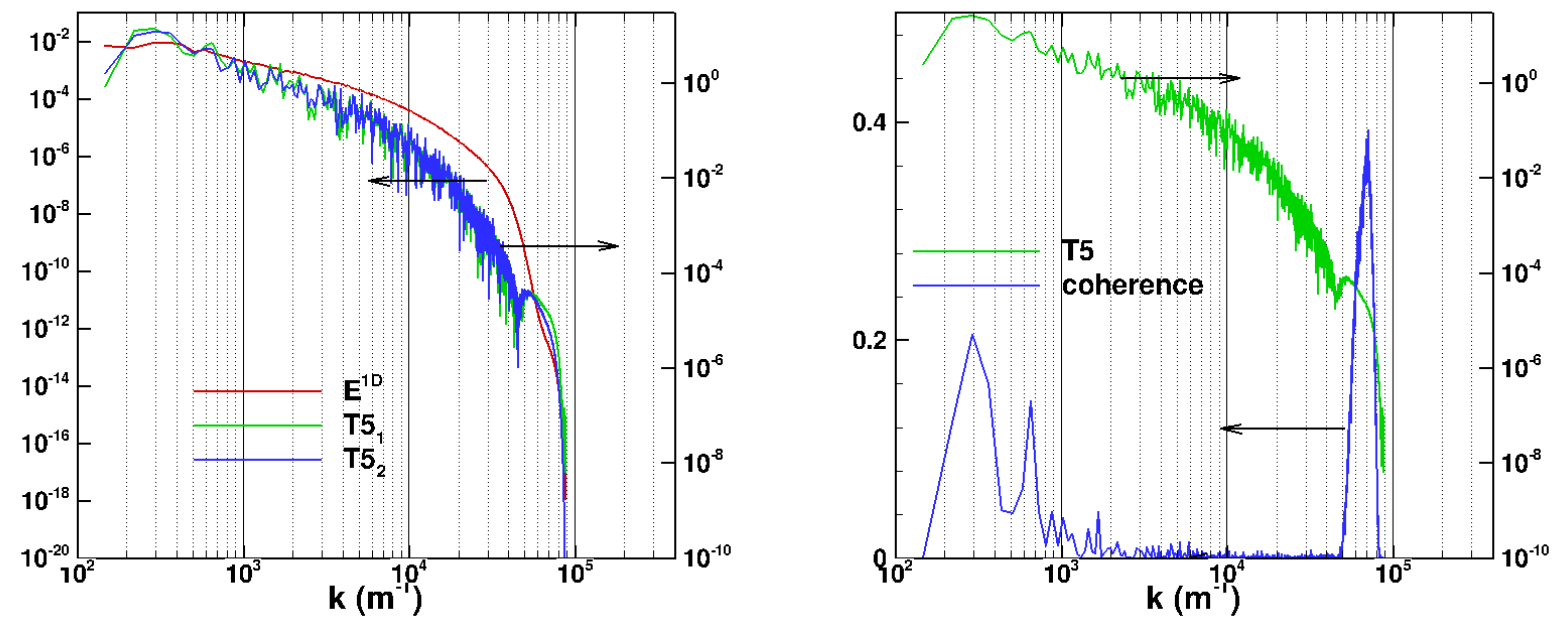

Figure 8. Plot on the left shows one-dimensional kinetic energy spectra for the Da+, $\widetilde{c}=0.1$ case, along with the pressure-velocity terms, $\mathrm{T} 5_{1}$ and $\mathrm{T} 5_{2}$ in Eq. 3.12. The plot on the right shows the total pressure-velocity term, T5, and the spectral coherence between $p^{\prime}$ and $u_{1}^{\prime \prime}$.

malised, it now appears that the location of the inflection in the kinetic energy spectrum coincides for a given Da. Furthermore this location is at $\left(k \delta_{L} / 2 \pi\right) \approx 5$ for the Da+ case and $\left(k \delta_{L} / 2 \pi\right) \approx 10$ for the Da- case, lending further credence to the hypothesis that the inflection arises due to the pressure-velocity term at the laminar flame scales. Further evidence of the contribution of this term is presented in Fig. 10 for all the cases. One can spot a direct correlation between both the magnitude and location of the 'spike' in this term at high wavenumber and the inflection in the corresponding kinetic energy spectra. It is worth emphasising, as is evident from Fig. 9, that the normalisation based on $\delta_{L}$, while improving the collapse in the viscous range, disrupts the collapse in the inertial range. This merely suggests that for an appropriate scaling to be valid over the entire wavenumber range it would have to involve both $\widetilde{\eta}$ and $\delta_{L}$. We envision suitably incorporating the Karlovitz number which, being related to the ratio of $\widetilde{\eta}$ and $\delta_{L}$, quantifies the extent of overlap (or lack thereof) between the viscous range and the reactive range. However, the derivation of the revised scaling is a topic for future study.

\subsection{Reacting scalar spectra}

In this section the auto-spectra of reactive scalars are interpreted based on the balance equation 3.16, which more generally, can be used to study both co-spectra and auto-spectra. The latter is often of interest in modelling methodologies that employ a 'laminar flamelet' hypothesis which attempt to capture the evolution of the entire chemical manifold through the evolution of a single, or sometimes two, reactive scalars. Figure 11 shows the one-dimensional auto-spectra of hydrogen and oxygen mass fractions for the Da- case at the three locations; $\widetilde{c}=0.1,0.5$ and 0.9 . In comparison to the kinetic energy spectra, the scalar spectra show a subtle difference; the curve corresponding to middle of the flame brush $(\tilde{c}=0.5)$ is higher than those corresponding to the flame brush edges $(\tilde{c}=0.1,0.9)$. This is a reflection of the scalar fluctuations peaking in the flame brush middle whereas the turbulent kinetic energy peaks near the unburnt side, as shown in Fig. 3 The hydrogen spectrum exhibits a shoulder, again in the high wavenumber reactive range, which is not so prominent for the oxygen spectrum. Curiously, for hydrogen, the 


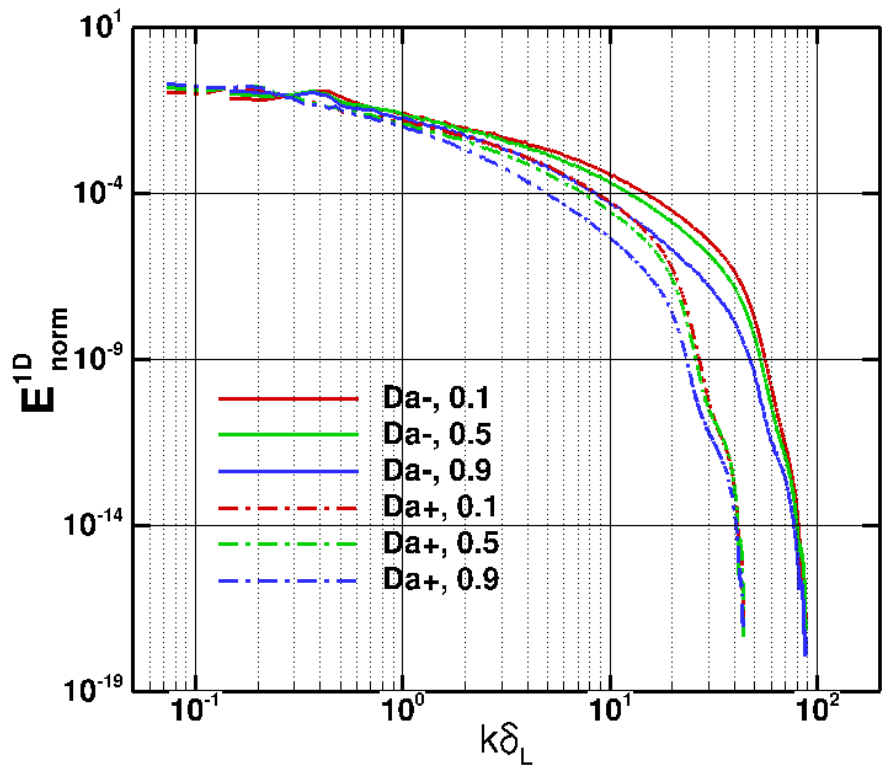

FiguRE 9. The one-dimensional turbulent kinetic energy spectra normalised using the laminar flame thickness as, $E_{\text {norm }} \equiv \widetilde{\epsilon}^{-2 / 3} \delta_{L}^{-5 / 3} E(k)$, plotted against the normalised wavenumber , $k \delta_{L}$,for all locations in the Da- and Da+ cases.
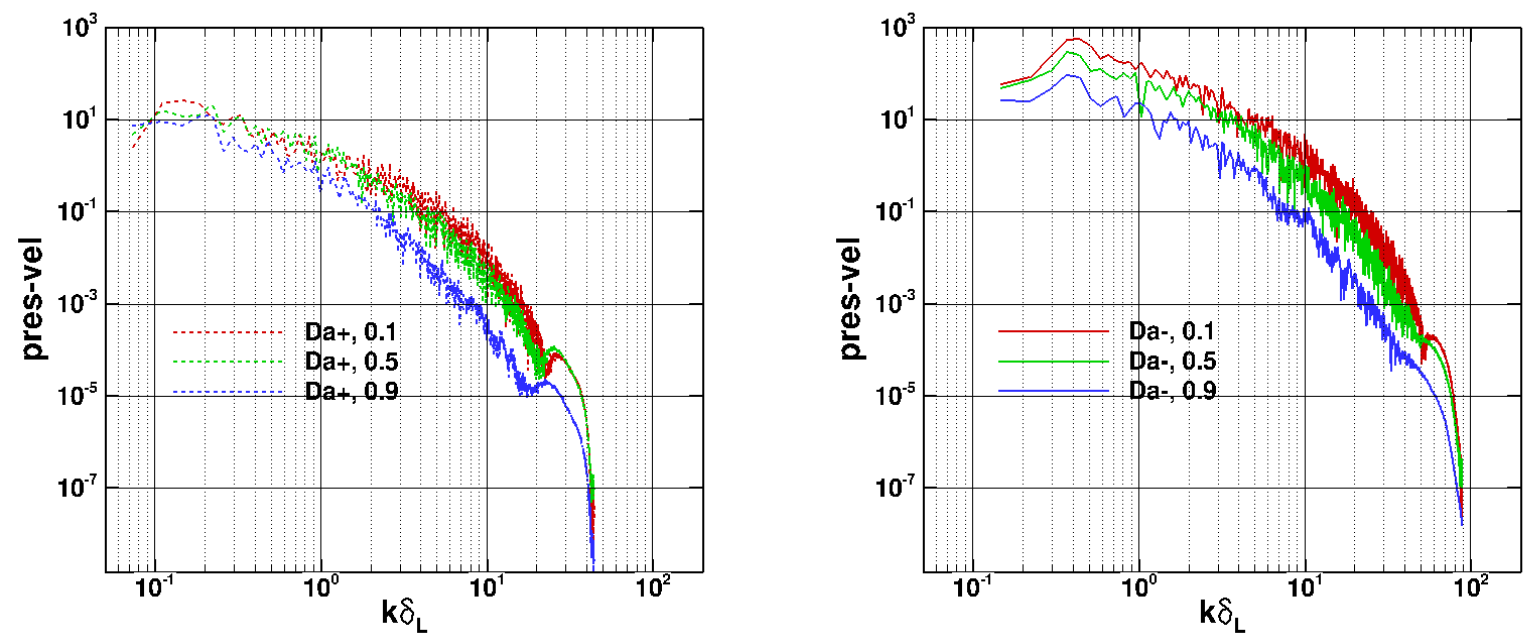

Figure 10. The total pressure-velocity term, T5 in Eq. 3.12, is plotted against the normalised wavenumber , $k \delta_{L}$, for the $\mathrm{Da}+$ (left) and Da- (right) cases.

shoulder exists at $\left(k \delta_{L} / 2 \pi\right) \approx 10$ which is the wavenumber where the pressure-velocity term has a signature. This is at least partly explained by the fact that for the current simulations the length scale corresponding to hydrogen reactions in the unstrained lam- 

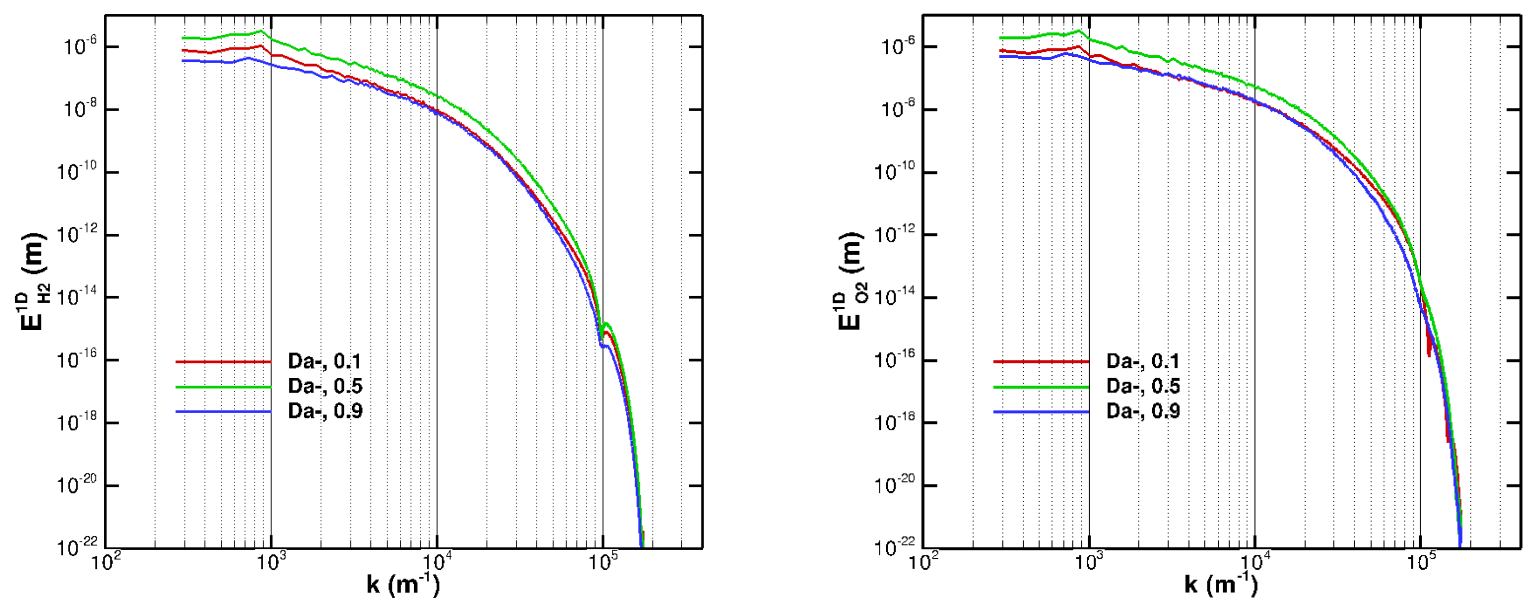

FiguRE 11. One dimensional auto-spectra of fluctuations of hydrogen (left) and oxygen (right) mass fractions for the Da- case at three locations; $\widetilde{c}=0.1,0.5$ and 0.9 .

inar flame is $3.6 \times 10^{-4} \mathrm{~m}$ which is very close to $\delta_{L}$. This thickness is estimated as the thickness of the hydrogen reaction-rate profile at $10 \%$ of its maximum.

We now consider the scaling for scalar spectra. The spectrum of a unity Schmidt number scalar is expected to have an inertial-convective wavenumber range where the spectral transfer is simply by convection with the well known Obukhov-Corrsin scaling,

$$
E_{\psi}(k) \sim \epsilon_{\psi} \epsilon^{-1 / 3} k^{-5 / 3},
$$

where $\epsilon_{\psi}$ is the scalar dissipation rate. It is only if the Schmidt number is sufficiently different than unity can one expect either a viscous-convective range $(\mathrm{Sc} \gg 1)$ or an inertial-diffusive range $(\mathrm{Sc} \ll 1)$. For the present case the Schmidt number for hydrogen is $\approx 0.2$ and that for oxygen is $\approx 0.8$ throughout the flame so it is unlikely that either limit would apply. As for the influence of chemical reactions the only guidance is from the analysis of Corrsin (1961) which yields, for a velocity-decoupled scalar undergoing a first-order chemical reaction, a scaling

$$
E_{\psi}(k) \sim A \overline{\psi^{\prime 2}} \epsilon^{-1 / 3} k^{-5 / 3} \exp \left(3 A \epsilon^{-1 / 3} k^{-2 / 3}\right),
$$

where $A$ is a time scale of the first-order chemical reactions. Corrsin's analysis shows the interesting behaviour that the influence of chemical reactions on the shape of the spectrum in the inertial-convective range diminishes as wavenumber increases. However, the applicability of this analysis too is unclear for the present study since the two central assumptions - scalar decoupled from velocity and first-order chemical reactions - are violated. Nonetheless, following Knaus \& Pantano (2009), we examine the ObukhovCorrsin scaling for the reactive scalars. Figure 12 shows the hydrogen spectra normalised using the Obukhov-Corrsin scale, $\widetilde{\eta}_{H_{2}} \equiv \widetilde{\eta} \mathrm{Sc}^{-3 / 4}$, as

$$
E_{\text {norm }} \equiv \widetilde{\epsilon}_{H 2}^{-1} \widetilde{\epsilon}^{1 / 3} \widetilde{\eta}_{H_{2}}^{-5 / 3} E_{H 2}
$$

and normalised using the laminar flame thickness (on the right) as

$$
E_{\text {norm }} \equiv \widetilde{\epsilon}_{H 2}^{-1} \widetilde{\epsilon}^{1 / 3} \delta_{L}^{-5 / 3} E_{H 2} \text {. }
$$

As in the case of the kinetic energy spectra, normalising with laminar flame thickness 

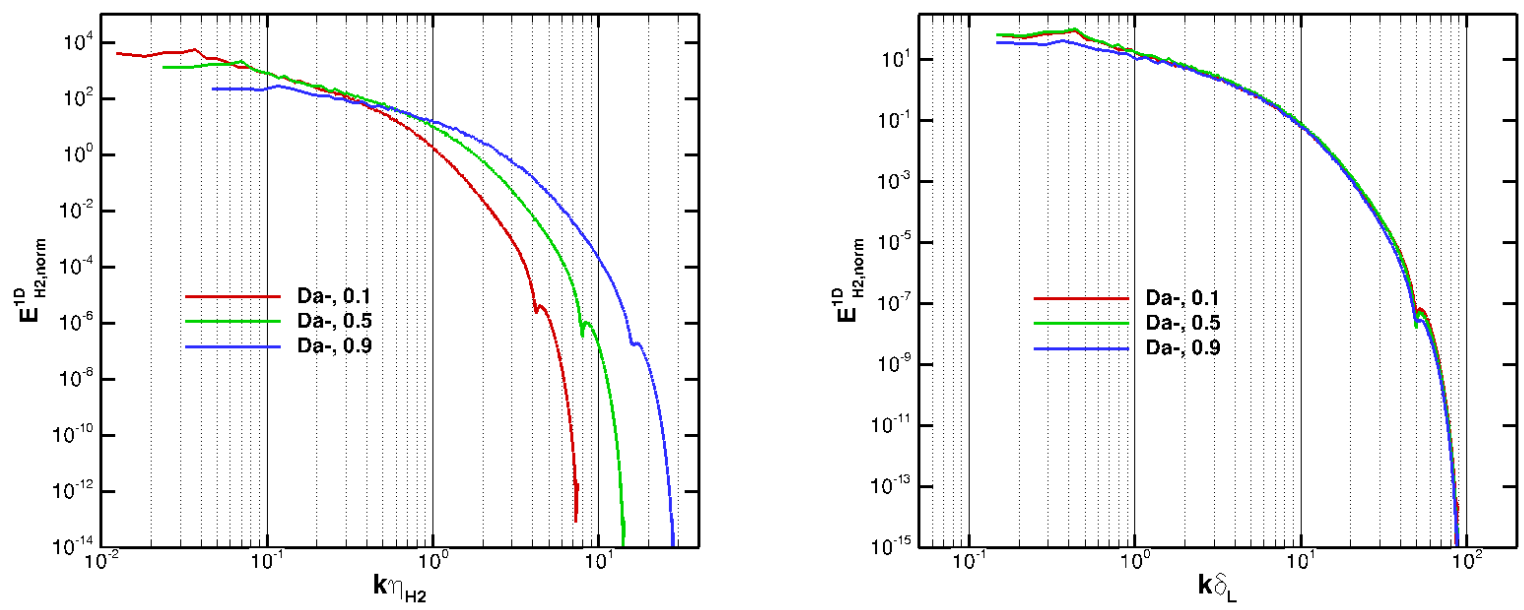

Figure 12. The one-dimensional hydrogen spectra normalised using the Obukhov-Corrsin scale as, $E_{\text {norm }} \equiv \widetilde{\epsilon}_{H 2}^{-1} \widetilde{\epsilon}^{1 / 3} \widetilde{\eta}_{H_{2}}^{-5 / 3} E_{H 2}$, (left) and using the laminar flame thickness as, $E_{\text {norm }} \equiv \widetilde{\epsilon}_{H 2}^{-1} \widetilde{\epsilon}^{1 / 3} \delta_{L}^{-5 / 3} E_{H 2}$, (right) plotted against the normalised wavenumber for the Dacase.
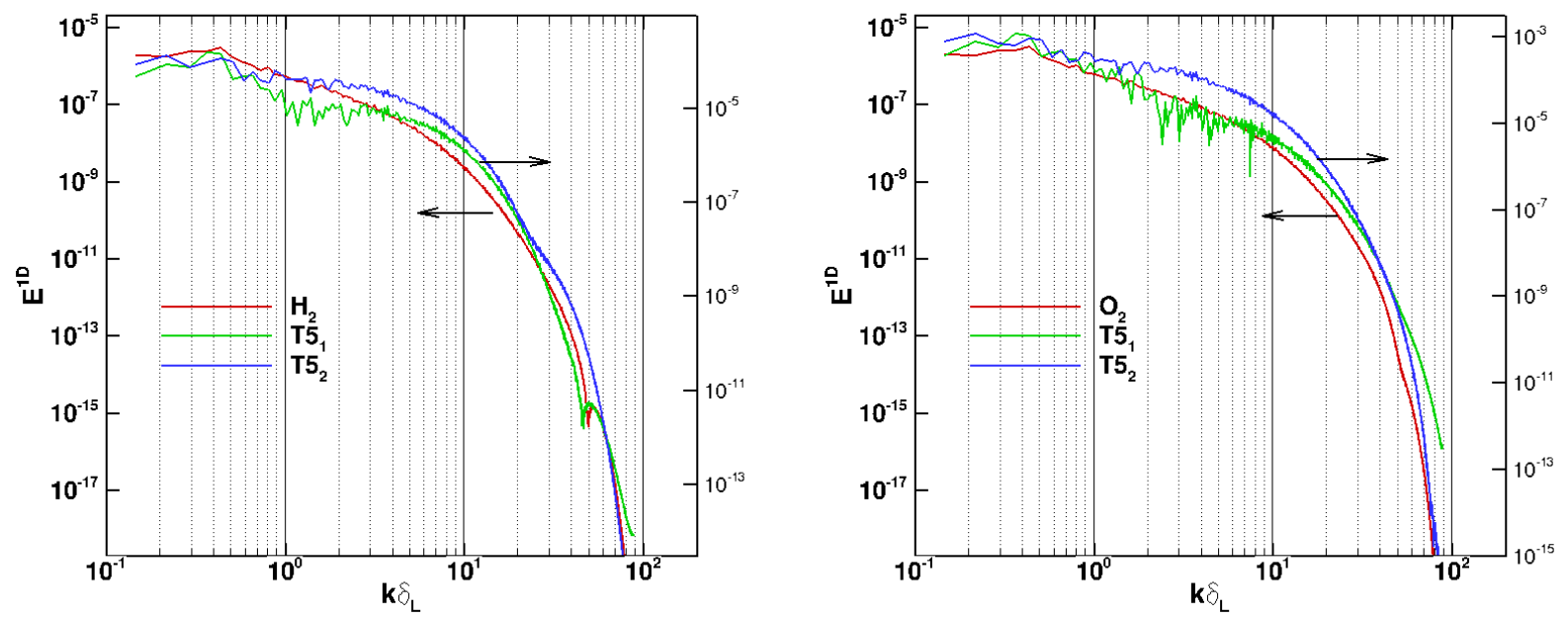

FIgURE 13. The reaction source terms, $\mathrm{T} 5_{1}$ and $\mathrm{T} 5_{2}$, in Eq. 3.16, for the hydrogen (left) and oxygen (right) auto-spectra are shown for the Da-, $\widetilde{c}=0.5$ case.

seems to give a better collapse compared to normalising with Obukhov-Corrsin scale. However, this collapse should not be treated as conclusive since the range of variation of the other normalising quantities is not very large between the three curves. Nonetheless, it is evident that the Obukhov-Corrsin scale does not collapse the spectra in the high wavenumber range which is consistent with the findings of Knaus \& Pantano (2009).

It was noted earlier that the reaction source term, T5 in Eq. 3.16, is analogous to the pressure-velocity term in Eq. 3.12. Both terms primarily represent the influence of 


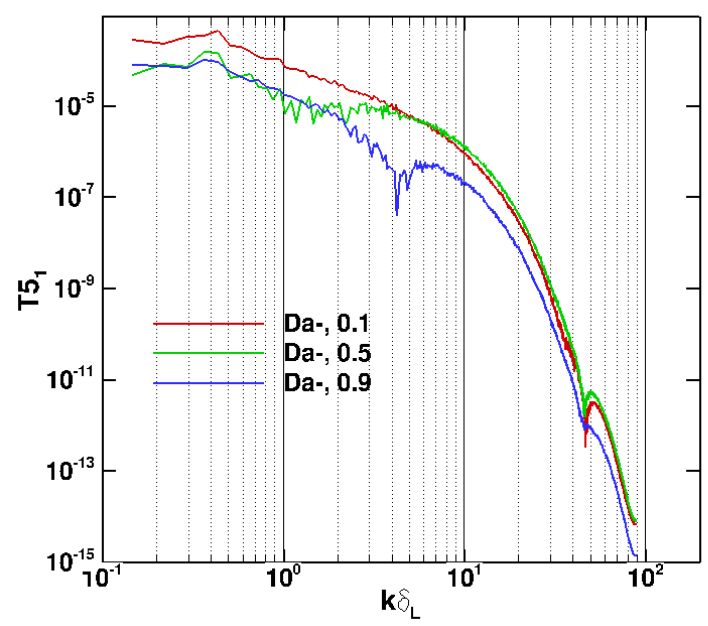

FiguRE 14. The reaction source term, T51, in Eq. 3.16, for the hydrogen auto-spectra are shown for the Da- case for the three $\widetilde{c}$ locations.

chemical reactions. The reaction source terms for the hydrogen and oxygen spectra are shown in Fig. 13. Again, the two terms, $\mathrm{T} 5_{1}$ and $\mathrm{T} 5_{2}$, are of similar magnitude and the presence (absence) of the shoulder in the auto-spectra for hydrogen (oxygen) correlates remarkably well with a corresponding strong (weak) contribution from one of the terms, $\mathrm{T} 5_{1}$. Curiously, the term $\mathrm{T} 5_{2}$ does not have such a spike. Furthermore, the bump in the hydrogen spectra is strongest for $\tilde{c}=0.5$, followed by 0.1 and 0.9 (see Fig.11) and this corresponds well with the magnitude of the $\mathrm{T} 5_{1}$ term for these three locations shown in Fig. 14. Figure 15 shows the hydrogen-oxygen co-spectra and the spectral coherence. The co-spectra, too, show a shoulder at exactly the same wavenumber. Furthermore, the spectral coherence distribution is quite interesting. The coherence between these two major species is high at the low wavenumber inertial range and it gradually becomes negligible, a consequence of the homogenisation of the scalar fluctuation correlations over the inertial range. However, the coherence abruptly increases at the wavenumber where the reactions are significant, clearly suggesting that the chemical reactions introduce thin fronts with a coherent variation of the species in a direction locally normal to the fronts.

\section{Concluding remarks}

The principal contributions of present study are twofold. First, we have presented a mathematical framework for a rigorous analysis of turbulent kinetic energy and scalar variances in spectral space for combusting flows with variable density. Although the primary focus was on turbulent reacting flows, this framework is equally applicable to non-reacting flows where density variation can be significant such as high Mach number turbulent flows. The cornerstone of the framework is a newly proposed density-weighted definition for two-point velocity and scalar correlation tensors that retain the essential properties of their incompressible counterparts while also being consistent with Favre averaged Reynolds-stresses and scalar co-variances. The density weighting formally leads to balance equations in wavenumber space for kinetic energy and scalar variance spectrum functions, hitherto only derived for constant-density flows (Hinze 1975). The balance 


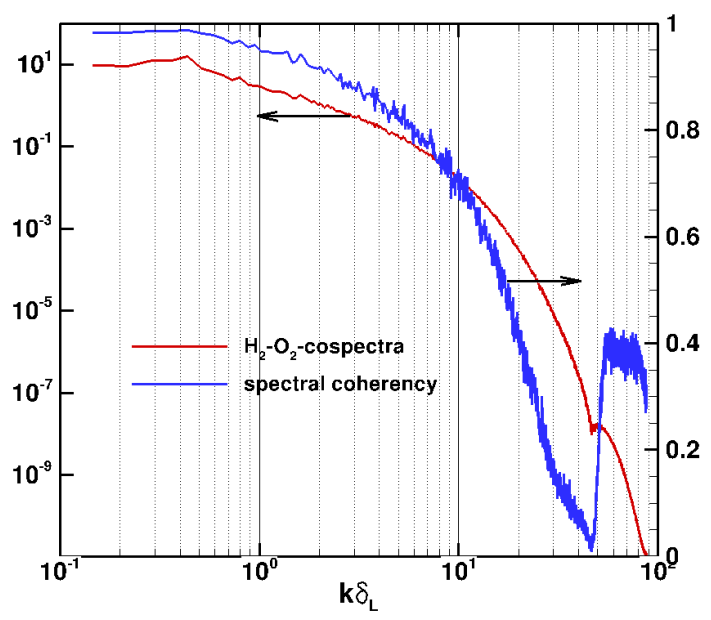

FiguRE 15. The one-dimensional co-spectra for fluctuating hydrogen-oxygen mass fractions, and their spectral coherence are shown for the Da-, $\widetilde{c}=0.5$ case.

equations clearly highlight the physics arising out of variable density such as the role of pressure-dilatation correlations in turbulent kinetic energy balance.

The second contribution of this study is the evidence of high wavenumber physics in turbulent reacting flows that result in spectra which do not adhere to classical incompressible scaling laws. High fidelity large Reynolds number direct numerical simulation data sets are used to construct kinetic energy spectra that clearly show that while Kolmogorov scaling may be applicable in the inertial range, the high wavenumber range should be construed as a 'diffusive-reactive' range where a proper scaling would have to incorporate a suitable length scale characteristic of chemical reactions. Likewise, the classical Obukhov-Corrsin scaling for near unity Schmidt number scalar spectra is not strictly applicable for reactive scalars particularly at high wavenumbers where chemical reactions occur. The DNS spectra substantiate the results from the spectral space balance analysis; pressure-dilatation correlations and reaction-scalar fluctuation correlations are high wavenumber sources of kinetic energy and scalar variance, respectively. However, these results are by no means conclusive since the DNS datasets have a somewhat limited scope. The Damköhler numbers of the DNS cases are relatively low $(\mathcal{O}(0.1))$ and vary only by a small factor across the data sets. Future work will focus on performing order of magnitude analyses on the spectral space balance equations to determine the relative contributions of various terms and the underlying scaling in different wavenumber ranges. The aim will be to assess the underlying assumptions of various sub-grid models and determine their regime of applicability for large eddy simulations. The framework can also enable a rigorous analysis of energy transfer in spectral space and study issues such as backscatter, another topic for future study. We will also attempt to glean spectra and assess the scaling by performing DNS over a much broader range of relevant parameters (Damköhler and Karlovitz numbers), and isolating parametric influences by utilizing simplified global chemical kinetics in future studies.

\section{Acknowledgements}

We are very grateful to the reviewers for their comments and suggestions which have 
considerably improved our paper, in particular to one of the referees who corrected some errors in our equations. Computational support for this project was supported by and this research used resources of the National Center for Computational Sciences at Oak Ridge National Laboratory, which is supported by the office of Science of the US Department of Energy under contract DE- AC05-00OR22725. The work at Sandia National Laboratories was supported by the Division of Chemical Sciences, Geosciences, and Biosciences, Office of Basic Energy Sciences of the US Department of Energy and by the US Department of Energy SciDAC Program. SNL is a multiprogramme laboratory operated by Sandia Corporation, a Lockheed Martin Company for the US DOE under Contract DE-AC04-94AL85000.

\section{Appendix A. Balance equation for velocity fluctuation $u_{i}^{\prime \prime}$}

We seek a balance equation for the velocity fluctuation about its Favre average, $u_{i}^{\prime \prime}$. The continuity equation, in its original and averaged forms is, respectively,

$$
\begin{aligned}
& \frac{\partial \rho}{\partial t}+\frac{\partial \rho u_{k}}{\partial x_{k}}=0, \\
& \frac{\partial \bar{\rho}}{\partial t}+\frac{\partial \bar{\rho} \widetilde{U}_{k}}{\partial x_{k}}=0 .
\end{aligned}
$$

Subtracting the latter from the former yields

$$
\frac{\partial \rho^{\prime}}{\partial t}+\frac{\partial \rho^{\prime} \widetilde{U}_{k}}{\partial x_{k}}+\frac{\partial \rho u_{k}^{\prime \prime}}{\partial x_{k}}=0
$$

On the other hand, the momentum equation can be expanded as

$$
\rho \frac{\partial u_{i}^{\prime \prime}}{\partial t}+\rho \frac{\partial \widetilde{U}_{i}}{\partial t}+\rho \widetilde{U}_{k} \frac{\partial \widetilde{U}_{i}}{\partial x_{k}}+\rho u_{k}^{\prime \prime} \frac{\partial \widetilde{U}_{i}}{\partial x_{k}}+\rho \widetilde{U}_{k} \frac{\partial u_{i}^{\prime \prime}}{\partial x_{k}}+\rho u_{k}^{\prime \prime} \frac{\partial u_{i}^{\prime \prime}}{\partial x_{k}}=-\frac{\partial\left(\bar{P}+p^{\prime}\right)}{\partial x_{i}}+\frac{\partial \tau_{i k}}{\partial x_{k}},
$$

where $\tau_{i k}$ is the viscous stress tensor. In the above, the conventional decompositions for density and pressure are used i.e. $\rho=\bar{\rho}+\rho^{\prime}$ and $p=\bar{P}+p^{\prime}$. Multiplying Eq. A 3 by $u_{i}^{\prime \prime}$ and adding to Eq. A 4 yields

$$
\begin{aligned}
\bar{\rho} \frac{\partial u_{i}^{\prime \prime}}{\partial t}+\frac{\partial \rho^{\prime} u_{i}^{\prime \prime}}{\partial t}+\rho \frac{\partial \widetilde{U}_{i}}{\partial t}+\rho \widetilde{U}_{k} \frac{\partial \widetilde{U}_{i}}{\partial x_{k}} & +\rho u_{k}^{\prime \prime} \frac{\partial \widetilde{U}_{i}}{\partial x_{k}}+\bar{\rho} \widetilde{U}_{k} \frac{\partial u_{i}^{\prime \prime}}{\partial x_{k}} \\
& +\frac{\partial \rho^{\prime} u_{i}^{\prime \prime} \widetilde{U}_{k}}{\partial x_{k}}+\frac{\partial \rho u_{i}^{\prime \prime} u_{k}^{\prime \prime}}{\partial x_{k}}=-\frac{\partial\left(\bar{P}+p^{\prime}\right)}{\partial x_{i}}+\frac{\partial \tau_{i k}}{\partial x_{k}} .
\end{aligned}
$$

It is straightforward to show that Eq. A 5, upon averaging and making use of Eq. A 2 and the relation

$$
\overline{\rho^{\prime} u_{i}^{\prime \prime}}=\overline{\rho u_{i}^{\prime \prime}}-\bar{\rho} \overline{u_{i}^{\prime \prime}}
$$

yields the averaged form of the momentum equation

$$
\bar{\rho} \frac{\partial \widetilde{U}_{i}}{\partial t}+\bar{\rho} \widetilde{U}_{k} \frac{\partial \widetilde{U}_{i}}{\partial x_{k}}+\frac{\partial \overline{\rho u_{i}^{\prime \prime} u_{k}^{\prime \prime}}}{\partial x_{k}}=-\frac{\partial \bar{P}}{\partial x_{i}}+\frac{\partial \bar{\tau}_{i k}}{\partial x_{k}} .
$$


Subtracting Eq. A 7 from Eq. A 5 and simplifying yields the desired density-weighted balance equation for $u_{i}^{\prime \prime}$

$$
\begin{aligned}
\frac{\partial \rho u_{i}^{\prime \prime}}{\partial t}+\rho^{\prime} \frac{\partial \widetilde{U}_{i}}{\partial t}+\rho^{\prime} \widetilde{U}_{k} \frac{\partial \widetilde{U}_{i}}{\partial x_{k}} & +\rho u_{k}^{\prime \prime} \frac{\partial \widetilde{U}_{i}}{\partial x_{k}} \\
& +\frac{\partial \rho u_{i}^{\prime \prime} \widetilde{U}_{k}}{\partial x_{k}}+\frac{\partial}{\partial x_{k}}\left(\rho u_{i}^{\prime \prime} u_{k}^{\prime \prime}-\overline{\rho u_{i}^{\prime \prime} u_{k}^{\prime \prime}}\right)=-\frac{\partial p^{\prime}}{\partial x_{i}}+\frac{\partial \tau_{i k}^{\prime}}{\partial x_{k}}
\end{aligned}
$$

The veracity of Eq. A 8 is easily verified. Multiplying the equation for $u_{i}^{\prime \prime}$ by $u_{j}^{\prime \prime}$, and the equation for $u_{j}^{\prime \prime}$ by $u_{i}^{\prime \prime}$, adding the two and averaging the results yields, when A 6 and A 7 are used, the exact balance equation for the Favre averaged Reynolds-stresses (Jones 1993):

$$
\begin{aligned}
\frac{\partial \bar{\rho} \widetilde{u_{i}^{\prime \prime} u_{j}^{\prime \prime}}}{\partial t} & +\frac{\partial \bar{\rho} \widetilde{U}_{k} \widetilde{u_{i}^{\prime \prime} u_{j}^{\prime \prime}}}{\partial x_{k}}+\widetilde{\bar{\rho} \widetilde{u_{i}^{\prime \prime} u_{k}^{\prime \prime}}} \frac{\partial \widetilde{U}_{j}}{\partial x_{k}}+\widetilde{\rho} \widetilde{u_{j}^{\prime \prime} u_{k}^{\prime \prime}} \frac{\partial \widetilde{U}_{i}}{\partial x_{k}}+\frac{\partial \overline{\rho u_{i}^{\prime \prime} u_{j}^{\prime \prime} u_{k}^{\prime \prime}}}{\partial x_{k}} \\
& =-\overline{u_{i}^{\prime \prime}} \frac{\partial \bar{P}}{\partial x_{j}}-\overline{u_{i}^{\prime \prime} \frac{\partial p^{\prime}}{\partial x_{j}}}-\overline{u_{j}^{\prime \prime}} \frac{\partial \bar{P}}{\partial x_{i}}-\overline{u_{j}^{\prime \prime} \frac{\partial p^{\prime}}{\partial x_{i}}}+\overline{u_{j}^{\prime \prime} \frac{\partial \tau_{i k}}{\partial x_{k}}}+\overline{u_{i}^{\prime \prime} \frac{\partial \tau_{j k}}{\partial x_{k}}}
\end{aligned}
$$

\section{Appendix B. Balance equation for scalar fluctuation $\psi^{\prime \prime}$}

The derivation for the scalar fluctuation equation proceeds along similar lines as for velocity. We start by expanding the balance equation for $\psi$ as:

$$
\rho \frac{\partial \psi^{\prime \prime}}{\partial t}+\rho \frac{\partial \widetilde{\psi}}{\partial t}+\rho \widetilde{U}_{k} \frac{\partial \widetilde{\psi}}{\partial x_{k}}+\rho u_{k}^{\prime \prime} \frac{\partial \widetilde{\psi}}{\partial x_{k}}+\rho \widetilde{U}_{k} \frac{\partial \psi^{\prime \prime}}{\partial x_{k}}+\rho u_{k}^{\prime \prime} \frac{\partial \psi^{\prime \prime}}{\partial x_{k}}=\dot{\omega}_{\psi}+\mathcal{D}_{\psi}
$$

where $\dot{\omega}_{\psi}$ is the reaction source term and $\mathcal{D}_{\psi}$ is the molecular diffusion term. Multiplying Eq. A 3 by $\psi^{\prime \prime}$ and adding the result to Eq. B 1 gives

$$
\begin{aligned}
\bar{\rho} \frac{\partial \psi^{\prime \prime}}{\partial t}+\frac{\partial \rho^{\prime} \psi^{\prime \prime}}{\partial t}+\rho \frac{\partial \widetilde{\psi}}{\partial t}+\rho \widetilde{U}_{k} \frac{\partial \widetilde{\psi}}{\partial x_{k}}+\rho u_{k}^{\prime \prime} \frac{\partial \widetilde{\psi}}{\partial x_{k}} & +\bar{\rho} \widetilde{U}_{k} \frac{\partial \psi^{\prime \prime}}{\partial x_{k}} \\
& +\frac{\partial \rho^{\prime} \widetilde{U}_{k} \psi^{\prime \prime}}{\partial x_{k}}+\frac{\partial \rho u_{k}^{\prime \prime} \psi^{\prime \prime}}{\partial x_{k}}=\dot{\omega}_{\psi}+\mathcal{D}_{\psi} .
\end{aligned}
$$

Averaging Eq. B 2 yields the exact balance equation for $\widetilde{\psi}$ :

$$
\bar{\rho} \frac{\partial \widetilde{\psi}}{\partial t}+\bar{\rho} \widetilde{U}_{k} \frac{\partial \widetilde{\psi}}{\partial x_{k}}+\frac{\partial \overline{\rho u_{k}^{\prime \prime} \psi^{\prime \prime}}}{\partial x_{k}}=\overline{\dot{\omega}}_{\psi}+\overline{\mathcal{D}}_{\psi}
$$

which, when subtracted from Eq. B 2 and simplified, yields the desired balance equation for $\psi^{\prime \prime}$ :

$$
\frac{\partial \rho \psi^{\prime \prime}}{\partial t}+\rho^{\prime} \frac{\partial \widetilde{\psi}}{\partial t}+\rho^{\prime} \widetilde{U}_{k} \frac{\partial \widetilde{\psi}}{\partial x_{k}}+\rho u_{k}^{\prime \prime} \frac{\partial \widetilde{\psi}}{\partial x_{k}}+\frac{\partial \rho \psi^{\prime \prime} \widetilde{U}_{k}}{\partial x_{k}}+\frac{\partial}{\partial x_{k}}\left(\rho u_{k}^{\prime \prime} \psi^{\prime \prime}-\overline{\rho u_{k}^{\prime \prime} \psi^{\prime \prime}}\right)=\dot{\omega}_{\psi}^{\prime}+\mathcal{D}_{\psi}^{\prime} .
$$

Note the similarity of Eq. B 4 to Eq. A 8.

\section{REFERENCES}

BAtchelor, G. K. 1959 Small-scale variation of convected quantities like temperature in turbulent fluid. Part 1. General discussion and the case of small conductivity. J. Fluid Mech. 5, 113-133. 
Batchelor, G. K., Howells, I. D. \& Townsend, A. A. 1959 Small-scale variation of convected quantities like temperature in turbulent fluid. Part 2 . The case of small conductivity. J. Fluid Mech. 5, 134-139.

Bilger, R. W. 2004 Some aspects of scalar dissipation. Flow Turb. Comb. 72, 93-114.

Bilger, R. W., Saetran, L. R. \& Krishnamoorthy, L. V. 1991 Reaction in a scalar mixing layer. J. Fluid Mech. 233, 211.

Chakraborty, N. \& Swaminathan, N. 2007 Influence of the Damköhler number on turbulence-scalar interaction in premixed flames. I. Physical insight. Phys. Fluids 19 (045103).

Chen, J. H., Choudhary, A., de Supinski, B., DeVries, M., Hawkes, E. R., Klasky, S., Liao, W. K., Ma, K. L., Mellor-Crummey, J., Podhorski, N., Sankaran, R., Shende, S. \& Yoo, C. S. 2009 Terascale direct numerical simulations of turbulent combustion using S3D. Comp. Sci. Disc. 2, 1-31.

Corrsin, S. 1961 The reactant concentration spectrum in turbulent mixing with a first order reaction. J. Flu. Mech 11, 407-416.

Dimotakis, P. E. 2005 Turbulent mixing. Annu. Rev. Fluid Mech. 37, 329-356.

Furukawa, J., Noguchi, Y., Hirano, T. \& Williams, F. A. 2002 Anisotropic enhancement of turbulence in large-scale, low-intensity turbulent premixed propane-air flames. J. Fluid Mech. 462, 209-243.

G. KosÁLY 1993 Frequency spectra of reactant fluctuations in turbulent flows. J. Fluid Mech. 246, 489-502.

Guttenfelder, W. A., King, G. B., Gore, J. P., Laurendeau, N. M. \& Renfro, M. W. 2003 Hydroxyl time-series measurements and simulations for turbulent premixed jet flames in the thickened preheat regime. Comb. Flame 135, 381-403.

Hawkes, E. R., Chatakonda, O., Kolla, H., Kerstein, A. R. \& Chen, J. H. 2012 A petascale direct numerical simulation study of the modelling of flame wrinkling for largeeddy simulations in intense turbulence. Comb. Flame (available online).

Hinze, J. O. 1975 Turbulence, 2nd edn. New York: McGraw-Hill.

Jones, W. P. 1993 Turbulence modelling and numerical solution methods for variable density and combusting flows. In Turbulent reacting flows (ed. P. A. Libby \& F. A. Williams), pp. 309-374. London: Academic Press.

Kariuki, J., Dawson, J. R. \& Mastorakos, E. 2012 Measurements in turbulent premixed bluff body flames close to blow-off. Comb. Flame 159, 2589-2607.

Kee, R. J., Dixon-Lewis, G., Warnatz, J., Coltrin, M. E. \& Miller, J. A. 1986 A Fortran computer code package for the evaluation of gas-phase multicomponent transport properties. Tech. Rep. SAND86-8246. Sandia National Laboratories.

Kee, R. J., Rupley, F. M., Meeks, E. \& Miller, J. A. 1996 Chemkin-III: a Fortran chemical kinetics package for the analysis of gas-phase chemical and plasma kinetics. Tech. Rep. SAND96-8216. Sandia National Laboratories.

Kennedy, C. A. \& CArpenter, M. H. 1994 Several new numerical methods for compressible shear-layer simulations. Applied Numerical Mathematics 14 (0), 397-433.

Kennedy, C. A., Carpenter, M. H. \& Lewis, R. M. 2000 Low-storage, explicit rungekutta schemes for the compressible navier-stokes equations. Applied Numerical Mathematics $35(0), 177-219$.

Knaus, R. \& Pantano, C. 2009 On the effect of heat release in turbulence spectra of nonpremixed reacting shear layers. J. Fluid Mech. 626, 67-109.

Krzywoblocki, M. Z. E. 1952 On the invariants in the turbulence in compressible viscous fluids. Journal of the Franklin Institute 254, 317-322.

Li, J., Zhao, Z., Kazarov, A. \& Dryer, F. L. 2004 An updated comprehensive kinetic model of hydrogen combustion. Int. J. Chem. Kinet. 36, 566-575.

PAssot, T. \& Pouquet, A. 1987 Numerical simulation of compressible homogeneous flows in the turbulent regime. J. Fluid Mech. 181, 441-466.

Poinsot, T. \& Lele, S. K. 1992 Boundary conditions for direct simulations of compressible viscous flow. Journal of Computational Physics 101, 104-129.

Pope, S. B. 2000 Turbulent Flows. Cambridge: Cambridge University Press.

Rogallo, R. S. 1981 Numerical experiments in homogeneous turbulence. NASA technical memorandum 81315. NASA Ames research center, Stanford, CA. 
Sarkar, S., Erlebacher, G., Hussaini, M. Y. \& Kreiss, H. O. 1991 The analysis and modelling of dilatational terms in compressible turbulence journal. J. Fluid Mech. 227, 473-493.

Swaminathan, N. \& Grout, R. W. 2006 Interaction of turbulence and scalar fields in premixed flames. Phys. Fluids 18 (045102).

Ulitsky, M. \& Collins, L. 1997 Application of the eddy damped quasi-normal Markovian spectral transport theory to premixed turbulent flames. Phys. Fluids 9 (3410).

Vaishnavi, P., Kronenburg, A. \& Pantano, C. 2008 On the spatial length scales of scalar dissipation in turbulent jet flames. J. Fluid Mech. 596, 103-132.

Wang, G., Karpetis, A. N. \& Barlow, R. S. 2007 Dissipation length scales in turbulent nonpremixed jet flames. Comb. Flame 148, 62-75.

Warhaft, Z. 2000 Passive scalars in turbulent flows. Ann. Rev. Flu. Mech. 32, 203-240.

Xia, Y., Liu, Y., Vaithianathan, T. \& Collins, L. R. 2010 Eddy damped quasinormal Markovian theory for chemically reactive scalars in isotropic turbulence. Phys. Fluids 22 (045103).

Yeung, P. \& Pope, S. B. 1989 J. Fluid Mech. 207, 531-586.

Yoo, C. S. \& IM, H. G. 2007 Characteristic boundary conditions for simulations of compressible reacting flows with multi-dimensional, viscous and reaction effects. Combust. Theory Modelling 11, 259-286.

Yoshizawa, A., Matsuo, Y. \& Mizobuchi, Y. 2013 A construction of the Reynolds-averaged turbulence transport equations in a variable-density flow, based on the concept of massweighted fluctuations. Phys. Fluids 25 (075105).

Zeman, O. 1991 On the decay of compressible isotropic turbulence. Phys. Fluids A 3, 951-955.

Zhang, S. \& Rutland, C. J. 1995 Premixed flame effects on turbulence and pressure-related terms. Comb. Flame 102, 447-461. 